\title{
Physics at a Higgsino factory
}

\author{
Howard Baer, ${ }^{a}$ Vernon Barger, ${ }^{b}$ Dan Mickelson, ${ }^{a}$ Azar Mustafayev ${ }^{c}$ and Xerxes Tata ${ }^{c}$ \\ ${ }^{a}$ Dept. of Physics and Astronomy, University of Oklahoma, \\ Norman, OK 73019, U.S.A. \\ ${ }^{b}$ Dept. of Physics, University of Wisconsin, \\ Madison, WI 53706, U.S.A. \\ ${ }^{c}$ Dept. of Physics and Astronomy, University of Hawaii, \\ Honolulu, HI 96822, U.S.A. \\ E-mail: baer@nhn.ou.edu, barger@pheno.wisc.edu, mickelso@nhn.ou.edu, \\ azar@phys.hawaii.edu, tata@phys.hawaii.edu
}

ABSTRACT: Naturalness arguments applied to supersymmetric theories imply a spectrum containing four light higgsinos $\widetilde{Z}_{1,2}$ and $\widetilde{W}_{1}^{ \pm}$with masses $\sim 100-300 \mathrm{GeV}$ (the closer to $M_{Z}$, the more natural). The compressed mass spectrum and associated low energy release from $\widetilde{W}_{1}$ and $\widetilde{Z}_{2}$ three-body decay makes higgsinos difficult to detect at LHC14, while the other sparticles might be heavy, and possibly even beyond LHC14 reach. In contrast, the International Linear $e^{+} e^{-}$Collider (ILC) with $\sqrt{s}>2 m$ (higgsino) would be a higgsino factory in addition to a Higgs boson factory and would serve as a discovery machine for natural SUSY! In this case, both chargino and neutralino production occur at comparable rates, and lead to observable signals above SM backgrounds. We examine two benchmark cases, one just beyond the LHC8 reach with $\widetilde{W}_{1}\left(\widetilde{Z}_{2}\right)-\widetilde{Z}_{1}$ mass gap of 15 (21) GeV, and a second more difficult case beyond even the LHC14 reach, where the mass gap is just $10 \mathrm{GeV}$, close to its minimum in models with no worse than $3 \%$ finetuning. The signal is characterized by low visible energy events together with $E_{T}$ in the one or two jets $+1 \ell$ channel from chargino production, and in the opposite sign, sameflavour, acoplanar dilepton channel from $\widetilde{Z}_{1} \widetilde{Z}_{2}$ production. For both cases, we find that the signal is observable above backgrounds from the usual $2 \rightarrow 2 \mathrm{SM}$ events and from $\gamma \gamma$ collisions with just a few $\mathrm{fb}^{-1}$ of integrated luminosity. We also show that with an integrated luminosity of $100 \mathrm{fb}^{-1}$, it should be possible to extract $\widetilde{W}_{1}$ and $\widetilde{Z}_{1}$ masses at $2-3 \%$ level from chargino events if the mass gap is $\geq 15 \mathrm{GeV}$, and neutralino masses at the sub-percent level from neutralino events. The latter should also allow a determination of $m_{\widetilde{Z}_{2}}-m_{\widetilde{Z}_{1}}$ at the $200 \mathrm{MeV}$ level. These measurements would point to higgsinos as the origin of new physics and strongly suggest a link to a natural origin for $W, Z$ and $h$ masses.

KeYwords: Supersymmetry Phenomenology

ARXiv EPRINT: 1404.7510 


\section{Contents}

1 Introduction: naturalness and light higgsinos 1

2 Two RNS benchmark points 4

$\begin{array}{lll}3 & \text { Sparticle production and decay at a higgsino factory } & 6\end{array}$

$\begin{array}{lll}3.1 & \text { Sparticle production } & 6\end{array}$

3.2 Higgsino decays 8

4 SUSY event generation $\quad 9$

5 Detection of higgsinos at a higgsino factory 10

$\begin{array}{ll}5.1 \text { Benchmark ILC1 at } \sqrt{s}=250 \mathrm{GeV} & 10\end{array}$

$\begin{array}{lll}5.1 .1 & \text { Chargino pair production } & 13\end{array}$

$\begin{array}{ll}\text { 5.1.2 Neutralino pair production } & 15\end{array}$

$\begin{array}{ll}5.2 \text { Benchmark ILC2 at } \sqrt{s}=340 \mathrm{GeV} & 19\end{array}$

$\begin{array}{ll}\text { 5.2.1 Chargino pair production for ILC2 } & 19\end{array}$

5.2.2 Neutralino pair production for ILC2 22

6 Conclusions 26

\section{Introduction: naturalness and light higgsinos}

Now that a (supposedly fundamental) scalar boson has been discovered with mass $m_{h}=$ $125.5 \pm 0.5 \mathrm{GeV}$ at LHC $[1,2]$, the puzzle is: why is it so light? It is well known that radiative corrections pull elementary scalar masses up to the mass of the heaviest particle to which the Higgs boson couples; e.g. to the GUT scale in the context of Grand Unified theories. Theories with supersymmetry softly broken at the weak scale have much better ultra-violet behaviour and so allow for the co-existence of the weak energy scale with $M_{\mathrm{GUT}}$. But so far, there is no sign of supersymmetric matter at LHC8 [3-8]. This latter issue has given rise to a supposed crisis in supersymmetric naturalness, and has lead some authors to question whether the simple picture of weak scale supersymmetry addresses the issue of naturalness in a satisfactory manner $[9,10]$.

Such a discussion depends on the measure of supersymmetric naturalness which is adopted and how it is applied. Here, we will adopt the quantity $\Delta_{E W}[11-17]$ that leads to a model-independent bound on the magnitude of the superpotential parameter $|\mu|$ which is 
the higgsino mass in most models. ${ }^{1}$ To construct $\Delta_{E W}$, we begin with the scalar potential minimization condition including radiative corrections:

$$
\frac{M_{Z}^{2}}{2}=\frac{m_{H_{d}}^{2}+\Sigma_{d}^{d}-\left(m_{H_{u}}^{2}+\Sigma_{u}^{u}\right) \tan ^{2} \beta}{\tan ^{2} \beta-1}-\mu^{2}
$$

and require that the observed value of $M_{Z}^{2}$ is obtained without large cancellations between terms on the right-hand-side. Since all entries in eq. (1.1) are defined at the weak scale, the electroweak fine-tuning measure is defined by,

$$
\Delta_{E W} \equiv \max _{i}\left|C_{i}\right| /\left(M_{Z}^{2} / 2\right)
$$

where $C_{H_{d}}=m_{H_{d}}^{2} /\left(\tan ^{2} \beta-1\right), C_{H_{u}}=-m_{H_{u}}^{2} \tan ^{2} \beta /\left(\tan ^{2} \beta-1\right)$ and $C_{\mu}=-\mu^{2}$. Also, $C_{\Sigma_{u}^{u}(k)}=-\Sigma_{u}^{u}(k) \tan ^{2} \beta /\left(\tan ^{2} \beta-1\right)$ and $C_{\Sigma_{d}^{d}(k)}=\Sigma_{d}^{d}(k) /\left(\tan ^{2} \beta-1\right)$, where $k$ labels the various loop contributions included in eq. (1.1). A low value of $\Delta_{E W} \lesssim 10-30$ means that all contributions to $M_{Z}$ (or equivalently, to $m_{h}$ ) are comparable to $M_{Z}$ (or $m_{h}$ ), i.e. no large cancellation between terms is required to generate the mass scale $M_{W}, M_{Z}, m_{h} \sim 100 \mathrm{GeV}$.

In order to generate low $\Delta_{E W} \sim 10-30,{ }^{2}$ from eq. (1.1) we see that:

- $|\mu| \sim 100-300 \mathrm{GeV}$, the lower the better

- $m_{H_{u}}^{2}$ is driven radiatively to small (usually negative) values, and

- $\Sigma_{u}^{u}\left(\tilde{t}_{1,2}\right) \lesssim 100-300 \mathrm{GeV}$.

Here, the lower end of the range of $|\mu|$ comes from the LEP2 bound on the chargino, while the upper end comes from requiring $\Delta_{E W} \lesssim 3 \%$. The last requirement can be met when $m_{\tilde{t}_{1}} \sim 1-2 \mathrm{TeV}$ with $m_{\tilde{t}_{2}} \sim 2-4 \mathrm{TeV}$ with large mixing due to a large value of $A_{t}$. A large trilinear SUSY breaking term $A_{t}$ suppresses both the $\tilde{t}_{1}$ and $\tilde{t}_{2}$ radiative corrections whilst lifting $m_{h}$ into the $125 \mathrm{GeV}$ regime $[11,13]$. The required low values of $\Sigma_{u}^{u}\left(\tilde{t}_{1,2}\right)$ mean the top-squarks cannot be too heavy: in this case, much above a few $\mathrm{TeV}$, but nevertheless likely too heavy to be revealed in LHC searches. This is in sharp contrast to expectations from previous analyses of natural SUSY models, and in accord with 1. a light Higgs boson mass $m_{h} \sim 125 \mathrm{GeV}, 2$. suppression of anomalous contributions to the $b \rightarrow s \gamma$ branching

\footnotetext{
${ }^{1}$ The authors of ref. $[15,17]$ argue that the commonly adopted "large log" measure which favors light third generation squarks $m_{\tilde{t}_{1,2}, \tilde{b}_{1}} \lesssim 500 \mathrm{GeV}$ neglects a variety of non-independent contributions, leading to over-estimates of fine-tuning by orders of magnitude. Once dependent terms are collected, this measure reduces to $\Delta_{E W}$. They also argue that the traditional $\Delta_{B G}$ measure of fractional change in $M_{Z}^{2}$ against fractional change in model parameters is highly model-dependent for multi-parameter SUSY theories but reduces to $\Delta_{E W}$ when all soft parameters are correlated and expressed in terms of a single parameter such as the gravitino mass $m_{3 / 2}$. See ref. $[15,17]$ for a detailed discussion. In contrast, in ref. [16] $\Delta_{E W}^{-1}$ is regarded only as an upper bound on the fine-tuning in any model with a specified spectrum. For the purposes of this paper, it does not matter which view one adopts. It is only important that a value $|\mu|$ close to $M_{Z}$ is necessary for low fine-tuning.

${ }^{2}$ The importance of low $|\mu| \sim M_{Z}$ was emphasized in ref. [18-20]. The first of Ref's. [21-24] also remarks that there be no large cancellation between $m_{H_{u}}^{2}$ and $\mu^{2}$. Ref's [25-32] also adopt weak scale fine-tuning for their discussion. Ref. [11] introduces $\Delta_{E W}$ including finite radiative corrections, and notes that large $A_{t}$ suppresses radiative corrections while lifting the value of $m_{h}$.
} 
fraction (which is in near agreement with the SM value) and 3. lack of a signal from light stops at LHC8. The gluino mass, which contributes radiatively to $m_{\tilde{t}_{1,2}}$, is also then bounded from above [33]. For $\Delta_{E W} \lesssim 10(30)$, then $m_{\tilde{g}} \lesssim 2 \mathrm{TeV}(4 \mathrm{TeV})[13]$.

Thus, spectra from low $\Delta_{E W}$ models are characterized by:

- two higgsino-like charginos, $\widetilde{W}_{1}^{ \pm}$and two higgsino-like neutralinos, $\widetilde{Z}_{1}$ and $\widetilde{Z}_{2}$ with $m_{\widetilde{Z}_{1}}<m_{\widetilde{Z}_{2}}$ and all with masses $\sim|\mu| \sim 100-300 \mathrm{GeV}$

- well-mixed top and bottom squarks in the few $\mathrm{TeV}$ regime,

- $m_{\tilde{g}} \lesssim 2-4 \mathrm{TeV}$ and

- first/second generation squarks and sleptons in the $5-30 \mathrm{TeV}$ regime ${ }^{3}$ consistent with at least a partial decoupling solution to the SUSY flavor, $C P$, gravitino and p-decay problems [35-38].

Models with such spectra have been described as radiatively-driven natural supersymmetry, or RNS, since the value of $m_{H_{u}}^{2}$ is radiatively driven to values close to $M_{Z}^{2}$. RNS spectra can be realized in the 2-extra-parameter non-universal Higgs models (NUHM2), but not in more constrained models such as mSUGRA/CMSSM. For the case of mSUGRA, while $\mu$ can become low in the $\mathrm{HB} / \mathrm{FP}$ region, the rather heavy top squarks $m_{\tilde{t}_{1,2}} \sim 7-15 \mathrm{TeV}$ produce large $\Sigma_{u}^{u}\left(\tilde{t}_{1,2}\right)$ leading again to substantial fine-tuning [12].

Light higgsinos can be produced at large rates at LHC8 and LHC14 [39]. However, the compressed higgsino spectrum leads to only small visible energy release from $\widetilde{W}_{1} \rightarrow \widetilde{Z}_{1} f \bar{f}^{\prime}$ and $\widetilde{Z}_{2} \rightarrow \widetilde{Z}_{1} f \bar{f}$ decays (where $f$ denotes SM fermions). LHC14 should probe gluinos with mass up to $m_{\tilde{g}} \sim 2 \mathrm{TeV}$, assuming an integrated luminosity of $\sim 1000 \mathrm{fb}^{-1}$. This means that LHC14 probes about half of the gluino mass range allowed by RNS [39]. A distinctive wino pair production signal from $p p \rightarrow \widetilde{W}_{2}^{ \pm} \widetilde{Z}_{4} X$ followed by $\widetilde{W}_{2} \rightarrow W \widetilde{Z}_{1,2}$ and $\widetilde{Z}_{4} \rightarrow W^{ \pm} \widetilde{W}_{1}^{\mp}$ leads to a novel same-sign diboson signature which, assuming gaugino mass unification, gives a somewhat better reach than the gluino pair production channel, and is distinctive in SUSY models with light higgsinos [40]. A possible signal from monojets recoiling against nearly invisible higgsino pairs can occur at the $1 \%$ level above QCD background for the high luminosity LHC14 [41-43]; this does not appear to us to be enough to claim a discovery potential, though a study [44] has suggested discovery might be possible by examining the soft-lepton daughters of $\widetilde{W}_{1}$ and $\widetilde{Z}_{2}$.

In this paper we examine the detectability of the light higgsinos of RNS at the International Linear Collider (ILC), a proposed $e^{+} e^{-}$collider $[45,48]$ designed to operate at $\sqrt{s} \sim 0.25-1 \mathrm{TeV}$, with an added capability of electron beam polarization. While such a machine is often touted as a Higgs boson factory due to the capacity to study the reaction $e^{+} e^{-} \rightarrow Z h$, for the case of models with light higgsinos that are required for naturalness, ${ }^{4}$ the ILC would also become a higgsino factory and a SUSY discovery machine[49]!

\footnotetext{
${ }^{3}$ Large first/second generation squark and slepton masses can again lead to violations of naturalness due to EW $D$-term contributions unless one of several conditions leading to degeneracy patterns within GUT multiplets etc. is respected [34].

${ }^{4}$ We assume that the superpotential $\mu$-term that enters via the scalar Higgs potential also makes the dominant contribution to the higgsino mass. This is the case in all models that we are aware of. However,
} 
We remark here that early pioneering studies were performed by the JLC group on mixed higgsino-wino type of chargino pair production where mass gaps were around $50 \mathrm{GeV}$ [50]. Additional studies incorporating cascade decays were performed in ref. [51], and in ref. $[52,53]$ chargino pair production in the hyperbolic branch/focus point region [18-24] was examined also with $\sim 40 \mathrm{GeV}$ mass gaps. Very recent studies include those in ref. [54, 55].

Recently, studies of higgsino pair production with mass gaps of order $1 \mathrm{GeV}$ have been performed [56]. In these studies, use is made of initial state photon radiation and exclusive one-or-two particle hadronic decays of the charginos which have large branching fractions because the $Q$-value is limited at the $\mathrm{GeV}$-level. These studies were relevant for stringmotivated high-scale gauge-mediation models where the very large gaugino masses lead to uncomfortably large values of $\Delta_{E W} \sim 275$ [57]. In the current paper, we examine the case of models with $\Delta_{E W} \sim 10-30$ where mass gaps of $10-20 \mathrm{GeV}$ are typical, and for which the techniques of ref. [56] are not needed.

In section 2, we present two RNS benchmark models labeled as ILC1 and ILC2. In section 3, we discuss sparticle production and decay for the two RNS benchmark models at a higgsino factory. In section 4, we present some details of our signal and background event generation calculations. In section 5, we discuss how ILC acts as a "natural SUSY" discovery machine for light higgsino pair production and show how it can make precision measurements of the associated sparticle masses and mass gaps. We conclude in section 6 .

\section{Two RNS benchmark points}

We select two benchmark points for our study of light higgsinos in RNS models. To generate spectra for models with low $\Delta_{E W}$, we use the Isasugra spectrum generator $[58,59]$ from Isajet 7.84 [60]. Isajet versions $\geq 7.83$ compute a value of $\Delta_{E W}$ for each SUSY spectrum. For both benchmark models, we input parameters from the NUHM2 model [61-67], as listed in table 1. Point ILC1 is similar to benchmark RNS2 of ref. [11], except a lower $\mu=115 \mathrm{GeV}$ value has been selected to yield a spectrum of light higgsinos which would already be accessible at ILC250 (ILC with $\sqrt{s}=250 \mathrm{GeV}$ ). The mass gaps for ILC1 are $m_{\widetilde{W}_{1}}-m_{\widetilde{Z}_{1}}=14.6 \mathrm{GeV}$ and $m_{\widetilde{Z}_{2}}-m_{\widetilde{Z}_{1}}=21.3 \mathrm{GeV}$. While safe from LHC8 bounds, gluino and also wino production will lead to observable signals at LHC14 [39].

We also examine a much more challenging case of benchmark ILC2 which will likely be beyond the reach of LHC14. This point is chosen from the RNS model-line with $\mu=$ $150 \mathrm{GeV}$ introduced in ref. [39], with $m_{1 / 2}$ adjusted to obtain as small a mass gap as possible, consistent with $\Delta_{E W}^{-1}$ of no more than $3 \%$. For this challenging case, the mass gaps are rather small, with $m_{\widetilde{W}_{1}}-m_{\widetilde{Z}_{1}}=10.2 \mathrm{GeV}$ and $m_{\widetilde{Z}_{2}}-m_{\widetilde{Z}_{1}}=9.7 \mathrm{GeV}$. This point is not accessible to ILC250, so that we examine the feasibility of detection at a centre-of-mass energy just below the top pair threshold.

in models without any additional singlet that couples to higgsinos, a SUSY-breaking higgsino mass term would be soft [46, 47], and the connection between the higgsino mass and the scalar Higgs potential is lost. See also ref. [33]. 


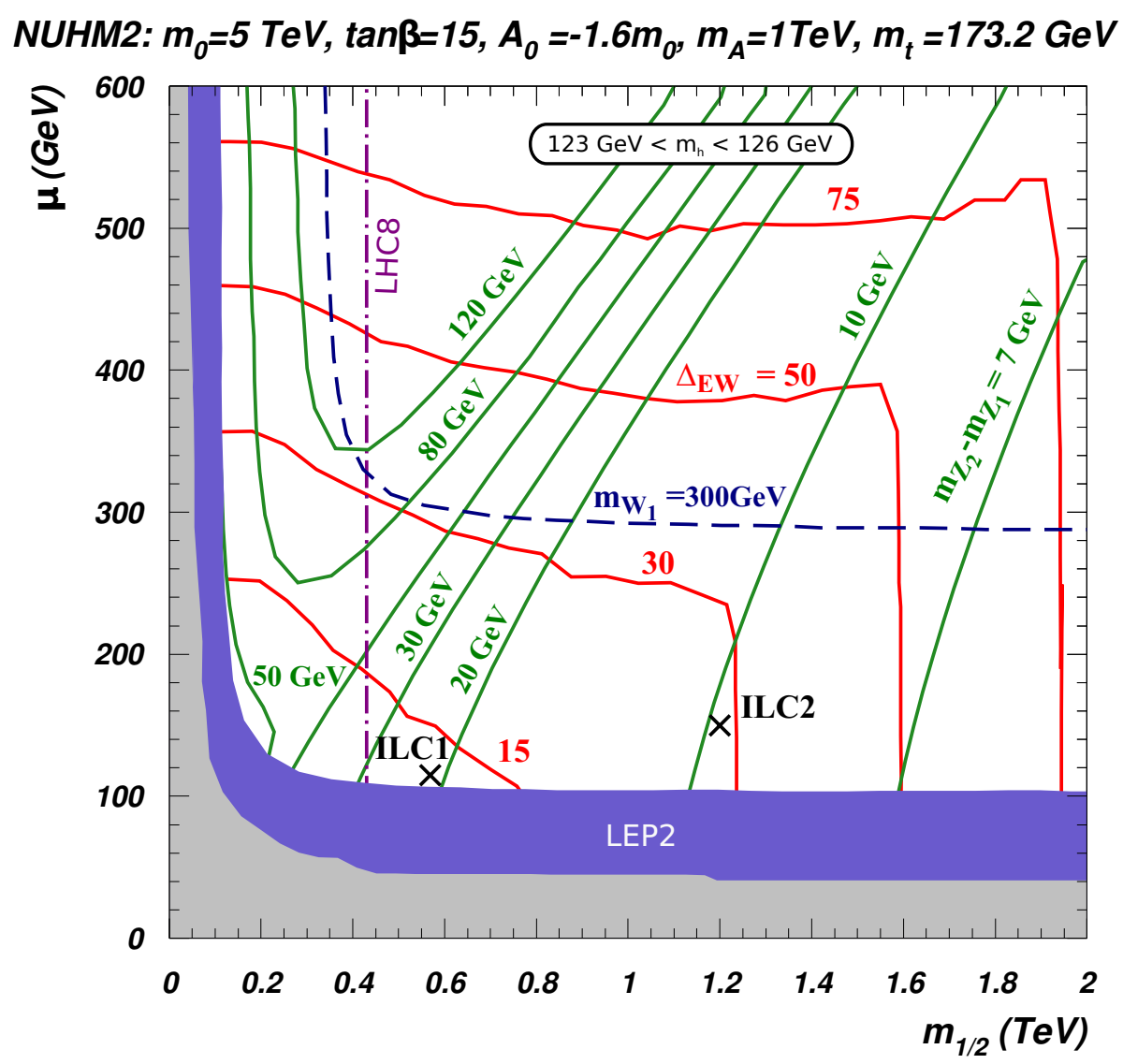

Figure 1. Contours of the mass gap (green curves) $m_{\widetilde{Z}_{2}}-m_{\widetilde{Z}_{1}}$ in the $m_{1 / 2}-\mu$ mass plane of the NUHM2 model for $m_{0}=5 \mathrm{TeV}, A_{0}=-1.6 m_{0}, \tan \beta=15$ and $m_{A}=1 \mathrm{TeV}$. The red curves show contours of $\Delta_{E W}$. The blue (gray) shaded regions are excluded by the absence of a chargino signal at LEP2 (LEP1). The region to the left of the dot-dashed line is excluded by the LHC8 limit $m_{\tilde{g}}>1.2 \mathrm{TeV}$, obtained assuming squarks are very heavy. The dashed line is where $m_{\widetilde{W}_{1}}=300 \mathrm{GeV}$. The crosses denote $\mu$ and $m_{1 / 2}$ values for ILC1 and ILC2 benchmark points. Note that the other parameters for ILC1 differ from those in the figure, but the mass difference is insensitive to these.

We stress that, within the RNS framework, $m_{\widetilde{Z}_{2}}-m_{\widetilde{Z}_{1}}=10 \mathrm{GeV}$ is close to the minimum of the mass gap if we require that $\Delta_{E W}^{-1}>3 \%$. This can be seen from figure 1 where we show the neutralino mass gap in the $m_{1 / 2}-\mu$ plane, with the other NUHM2 parameters fixed at the same values as for the ILC2 case. We see from the figure that the mass gap ranges from about $10 \mathrm{GeV}$ (for large $m_{1 / 2}$, where the $\Delta_{E W}$ contours become vertical because the top squarks become too heavy) to over $100 \mathrm{GeV}$ in the region where the gaugino and higgsino states are strongly mixed. For these large mass gaps, LHC experiments should be awash in clean multilepton signals from electroweak-ino production, including signals from $\widetilde{W}_{2}$ and $\widetilde{Z}_{4}$ events. ${ }^{5}$ Our concern here, of course, is the difficult region with a small mass gaps $m_{\widetilde{Z}_{2}}-m_{\widetilde{Z}_{1}}$ and $m_{\widetilde{W}_{1}}-m_{\widetilde{Z}_{1}}$ where there may well be no detectable

\footnotetext{
${ }^{5}$ Within the RNS framework where gaugino mass parameters are assumed to be unified, this region is excluded by the LHC gluino search. Electroweak-ino production can nonetheless serve to probe more general models.
} 
signals even at LHC14. To the extent that the difficulty of extracting ILC SUSY signals (without using kinematic properties particular to exclusive chargino decays [56]) increases with decreasing mass gap, the ILC2 point represents nearly the most challenging case that we may encounter in our examination of linear colliders as a definitive probe of naturalness.

We list at the bottom of table 1 the neutralino relic density, some $B$-decay branching fractions and WIMP detection rates along with the value of $\Delta_{E W}$. WIMP detection sensitivities should be multiplied by a factor $\xi \equiv \Omega_{\widetilde{Z}_{1}} h^{2} / 0.12$ since the higgsino-like WIMPs could make up only a fraction of the local DM density [68], whilst e.g. axions might make up the remainder $[69,70]$.

\section{Sparticle production and decay at a higgsino factory}

\subsection{Sparticle production}

In figure 2, we show sparticle and Higgs boson production rates for unpolarized beams at the ILC versus $\sqrt{s}$ for the ILC1 benchmark point. Rates were computed at leading order, with leading order spectra, using formulae from ref. [71]. Also shown for comparison is the rate for muon pair production. We see that around $\sqrt{s} \sim 220-230 \mathrm{GeV}$, the threshold for production of $Z h, \widetilde{W}_{1}^{ \pm} \widetilde{W}_{1}^{\mp}$ and $\widetilde{Z}_{1} \widetilde{Z}_{2}$ is crossed so that production rates rise rapidly. Whereas one might expect ILC at these energies to be a Higgs boson factory, we see that ILC would also be a higgsino factory, where the higgsino pair production rates exceed $Z h$ production by factors of 5-10! While the higgsino decay debris may be too soft to be picked out above SM backgrounds at LHC, the clean environment of a linear $e^{+} e^{-}$collider allows for straightforward discovery, as discussed in section 5. Thus, while radiatively-driven natural SUSY might well elude LHC searches, it cannot elude searches at ILC provided that $\sqrt{s}>2 m$ (higgsino).

While the reactions $e^{+} e^{-} \rightarrow \widetilde{W}_{1}^{+} \widetilde{W}_{1}^{-}$and $e^{+} e^{-} \rightarrow \widetilde{Z}_{1} \widetilde{Z}_{2}$ will be the first sparticle production processes accessed at ILC250, the discovery prospects do not end there. As $\sqrt{s}$ is increased beyond $2 m$ (higgsino), further thresholds will be passed, including those for $\widetilde{Z}_{2} \widetilde{Z}_{3}, \widetilde{W}_{1} \widetilde{W}_{2}$ and $\widetilde{Z}_{2} \widetilde{Z}_{4}$ production. These occur at somewhat lower but still measureable rates. Even reactions with much lower production rates - such as $\widetilde{Z}_{2} \widetilde{Z}_{2}, \widetilde{Z}_{1} \widetilde{Z}_{3}, \widetilde{Z}_{3} \widetilde{Z}_{3}$ and $\widetilde{Z}_{3} \widetilde{Z}_{4}$ - might ultimately be detectable, depending on the machine energy and integrated luminosity that is ultimately attained.

In figure 3 , we show the $\widetilde{W}_{1}^{+} \widetilde{W}_{1}^{-}$and $\widetilde{Z}_{1} \widetilde{Z}_{2}$ production rates for the ILC1 benchmark case at $\sqrt{s}=250 \mathrm{GeV}$, but as a function of the electron beam polarization $P_{L}\left(e^{-}\right)$. Here and in the rest of the paper, we assume that the positron beam is unpolarized, i.e. $P_{L}\left(e^{+}\right)=0$. Whereas $\widetilde{W}_{1}^{+} \widetilde{W}_{1}^{-}$production has the largest rate for unpolarized beams $\left(P_{L}\left(e^{-}\right)=0\right)$, for the case of right polarized electron beam, $\sigma\left(\widetilde{W}_{1}^{+} \widetilde{W}_{1}^{-}\right)$diminishes by a factor of about 4 and instead $\sigma\left(\widetilde{Z}_{1} \widetilde{Z}_{2}\right)$, which is much less sensitive to beam polarization, is dominant. The comparable rates (within an order of magnitude) for both both chargino and neutralino pair production (solid curves), together with the relatively mild polarization is characteristic of the production of higgsino-like charginos and neutralinos. For wino-like gauginos in the kinematically accessible range, chargino production would occur at a high rate, but neutralino pair production would be strongly suppressed because $\mathrm{SU}(2)_{L} \times \mathrm{U}(1)_{Y}$ gauge 


\begin{tabular}{|c|c|c|}
\hline parameter & ILC1 & ILC2 \\
\hline$m_{0}$ & 7025.0 & 5000 \\
\hline$m_{1 / 2}$ & 568.3 & 1200 \\
\hline$A_{0}$ & -11426.6 & -8000 \\
\hline $\tan \beta$ & 10 & 15 \\
\hline$\mu$ & 115 & 150 \\
\hline$m_{A}$ & 1000 & 1000 \\
\hline$m_{\tilde{g}}$ & 1563.5 & 2832.6 \\
\hline$m_{\tilde{u}_{L}}$ & 7021.3 & 5440.4 \\
\hline$m_{\tilde{u}_{R}}$ & 7254.2 & 5565.6 \\
\hline$m_{\tilde{e}_{R}}$ & 6758.6 & 4817.1 \\
\hline$m_{\tilde{t}_{1}}$ & 1893.3 & 1774.3 \\
\hline$m_{\tilde{t}_{2}}$ & 4919.4 & 3877.9 \\
\hline$m_{\tilde{b}_{1}}$ & 4959.2 & 3902.8 \\
\hline$m_{\tilde{b}_{2}}$ & 6893.3 & 5204.5 \\
\hline$m_{\tilde{\tau}_{1}}$ & 6656.6 & 4652.3 \\
\hline$m_{\tilde{\tau}_{2}}$ & 7103.1 & 5072.5 \\
\hline$m_{\tilde{\nu}_{\tau}}$ & 7114.0 & 5078.7 \\
\hline$m_{\widetilde{W}_{2}}$ & 513.0 & 1017.5 \\
\hline$m_{\widetilde{W}_{1}}$ & 117.3 & 158.3 \\
\hline$m_{\widetilde{Z}_{4}}$ & 524.2 & 1031.1 \\
\hline$m_{\widetilde{Z}_{3}}$ & 267.0 & 538.7 \\
\hline$m_{\widetilde{Z}_{2}}$ & 124.0 & 157.8 \\
\hline$m_{\widetilde{Z}_{1}}$ & 102.7 & 148.1 \\
\hline$m_{h}$ & 125.3 & 125.4 \\
\hline$\Omega_{\widetilde{Z}_{1}}^{s t d} h^{2}$ & 0.009 & 0.007 \\
\hline$B F(b \rightarrow s \gamma) \times 10^{4}$ & 3.3 & 3.3 \\
\hline$B F\left(B_{s} \rightarrow \mu^{+} \mu^{-}\right) \times 10^{9}$ & 3.8 & 3.9 \\
\hline$\sigma^{S I}\left(\widetilde{Z}_{1} p\right)(\mathrm{pb})$ & $1.3 \times 10^{-8}$ & $2.9 \times 10^{-9}$ \\
\hline$\Delta_{E W}$ & 13.9 & 28.5 \\
\hline
\end{tabular}

Table 1. NUHM2 input parameters and masses in $\mathrm{GeV}$ units for the two radiatively-driven natural SUSY benchmark points introduced in the text. We take $m_{t}=173.2 \mathrm{GeV}$

symmetry forbids couplings of the $Z$ and $\gamma$ to both binos and (neutral) winos. ${ }^{6}$ This can be seen in the dashed curve in figure 3 which shows the cross section for $\widetilde{W}_{1} \widetilde{W}_{1}$ production for the ILC1 NUHM2 model point except that $m_{1 / 2}$ and $\mu$ are now chosen so that the weak scale values of $M_{2}$ and $\mu$ are essentially exchanged. In this case, the masses of the wino-like

\footnotetext{
${ }^{6}$ This assumes that the selectron is heavy so that neutralino production via $t$-channel selectron production is negligible. Neutralino production via $t$-channel selectron exchange also yields a large rate for $\widetilde{Z}_{2} \widetilde{Z}_{2}$ production, so should be readily distinguishable since there would be events also in the $4 \ell, 2 \ell 2 j$ and $4 j+E_{T}$ channels. The angular distributions of the neutralinos will also be different if $t$-channel exchange contributions are significant.
} 


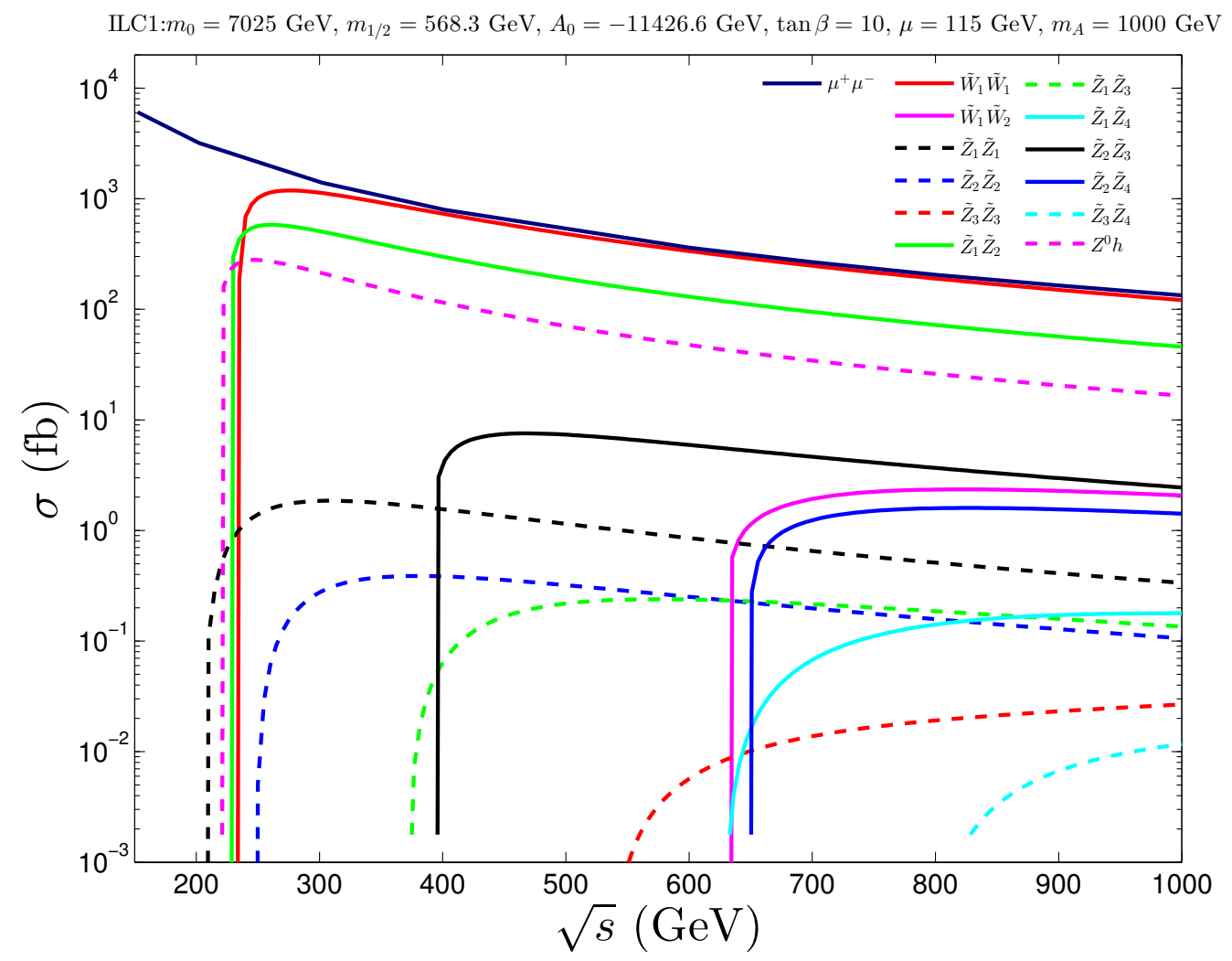

Figure 2. Sparticle production cross sections vs. $\sqrt{s}$ for unpolarized beams at an $e^{+} e^{-}$collider for the ILC1 benchmark point listed in table 1.

$\widetilde{W}_{1}$ and $\widetilde{Z}_{2}$ is about the same as for the higgsinos of the ILC1 point. The neutralino-pair production cross sections for this wino-like case are below $0.1 \mathrm{fb}$ and do not show up in this frame. This observation will be important in section 5 where we describe our analysis. The polarization dependence of the chargino pair production cross section provides an independent handle that may enable us to argue the higgsino-like nature of the charginos of the ILC1 point. For a right-handed electron beam the amplitude for charged wino pair production is suppressed by a factor of $M_{W}^{2} / s$ relative to that for charged higgsino pair production, accounting for the strong drop of the dashed curve at $P_{L}\left(e^{-}\right)=-1$.

\subsection{Higgsino decays}

Since the inter-higgsino mass gaps are so small, for the case of RNS one expects the following three-body decays to be dominant:

$$
\begin{aligned}
\widetilde{W}_{1}^{-} & \rightarrow \widetilde{Z}_{1} f \bar{f}^{\prime} \\
\widetilde{Z}_{2} & \rightarrow \widetilde{Z}_{1} f \bar{f},
\end{aligned}
$$

where the $f$ stand for SM fermions. Despite the larger phase space suppression for the three body decays, the branching fraction for the loop decay $\widetilde{Z}_{2} \rightarrow \widetilde{Z}_{1} \gamma$ is still small because of 


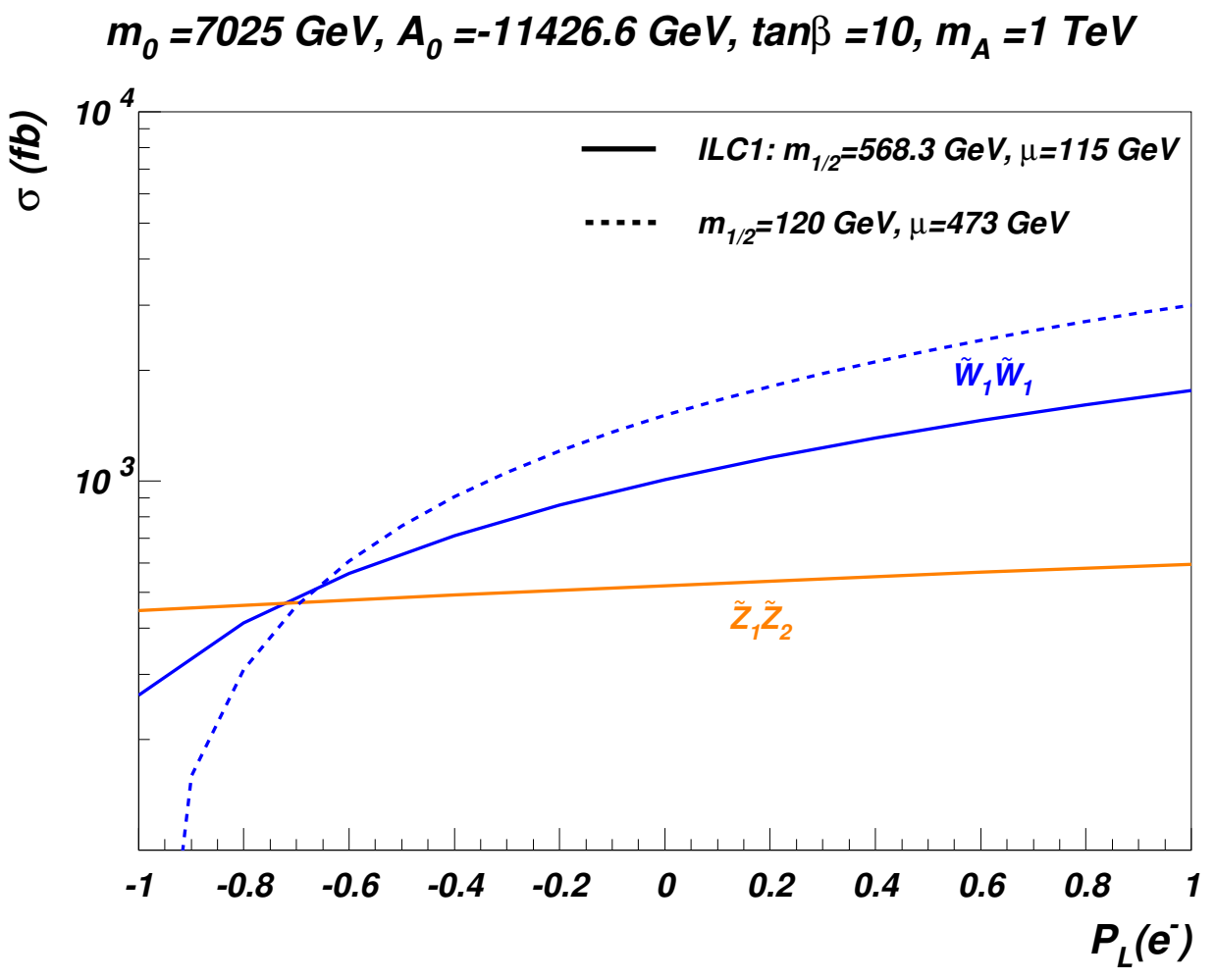

Figure 3. Sparticle production cross sections vs. $P_{L}\left(e^{-}\right)$at an $e^{+} e^{-}$collider for the ILC1 benchmark point with $\sqrt{s}=250 \mathrm{GeV}$. The positrons are taken to be unpolarized. For comparison, we show a point with a wino-like chargino of similar mass. For the wino-like case with $m_{1 / 2}=120 \mathrm{GeV}$, then the $\sigma\left(e^{+} e^{-} \rightarrow \widetilde{Z}_{1} \widetilde{Z}_{2}\right) \sim 0.1 \mathrm{fb}$, while $\sigma\left(\widetilde{Z}_{2} \widetilde{Z}_{2}\right)$ is even smaller, and so is far below the cross section values shown.

the large $Z \widetilde{Z}_{1} \widetilde{Z}_{2}$ coupling [72-75]. Moreover, squark and slepton masses are expected very large within the RNS framework, and the $\widetilde{W}_{1}$ and $\widetilde{Z}_{2}$ three-body decay amplitudes are dominated by $W^{*}$ and $Z^{*}$ exchange, respectively. The branching fractions into specific modes will thus closely follow the corresponding $W$ and $Z$ decay branching fractions, i.e. we obtain $B\left(\widetilde{W}_{1}^{-} \rightarrow \widetilde{Z}_{1} e^{-} \bar{\nu}_{e}\right) \simeq 11 \%, B\left(\widetilde{Z}_{2} \rightarrow e^{+} e^{-} \widetilde{Z}_{1}\right) \simeq 3 \%$, etc..

\section{SUSY event generation}

Within the RNS framework, higgsino pair production at the ILC will be signalled by events with low visible energy from the relatively soft daughter leptons and jets from $\widetilde{W}_{1}$ and $\widetilde{Z}_{2}$ decays, and modest $E_{T}$. We need, therefore, to pay particular attention to SM sources of low visible energy events. Since the bulk of $2 \rightarrow 2$ events lead to large visible energy, the most important backgrounds come from two photon processes, $e^{+} e^{-} \rightarrow e^{+} e^{-} f \bar{f}$, where the energetic final state electron and positron are lost down the beam-pipe, and the visible energy in the detector arises only from $f$ and $\bar{f}$. 
We use ISAJET v7.84 for our SUSY event simulation as well as simulation of $2 \rightarrow 2$ and $\gamma \gamma$-induced SM backgrounds. The $2 \rightarrow 2$ SM background sources include

$$
e^{+} e^{-} \rightarrow f \bar{f}, W^{+} W^{-} \text {and } Z^{0} Z^{0}
$$

while $\gamma \gamma$ processes include,

$$
\gamma \gamma \rightarrow \tau^{+} \tau^{-}, \quad c \bar{c} \text { and } b \bar{b}
$$

The reaction $e^{+} e^{-} \rightarrow Z h$ is included in the signal sample, but plays no role here.

We use the Isajet toy calorimeter covering $-4<\eta<4$ with cell size $\Delta \eta \times \Delta \phi=$ $0.05 \times 0.05$. Energy resolution for electromagnetic and hadronic depositions is taken to be $\Delta E_{e m} / E_{e m}=0.15 / \sqrt{E_{e m}} \oplus 1 \%$ and $\Delta E_{h} / E_{h}=0.5 / \sqrt{E_{h}} / E_{h} \oplus 2 \%$, respectively (where $\oplus$ denotes addition in quadrature). Jets are found using fixed cones of size $R=$ $\sqrt{\Delta \eta^{2}+\Delta \phi^{2}}=0.6$ using the ISAJET routine GETJET (modified for clustering on energy rather than transverse energy). Clusters with $E>5 \mathrm{GeV}$ and $\mid \eta($ jet $) \mid<2.5$ are labeled as jets. Muons and electrons are classified as isolated if they have $E>5 \mathrm{GeV},|\eta(\ell)|<2.5$, and the visible activity within a cone of $R=0.5$ about the lepton direction is less than $\min \left(\frac{E_{\ell}}{10}, 1 \mathrm{GeV}\right)$.

Our production reactions are run using electron PDFs which include a convolution of bremsstrahlung [76] and beamstrahlung [77, 78] contributions as described in ref. [53]. For $\sqrt{s}=250 \mathrm{GeV}$, we use beamstrahlung parameter $\Upsilon=0.02$ and for $\sqrt{s}=340 \mathrm{GeV}$, we use $\Upsilon=0.03$. For both cases, we use the beam bunch length $\sigma_{z}=0.3 \mathrm{~mm}$ [79].

For processes with low visible energy, the two-photon processes $\gamma \gamma \rightarrow f \bar{f}$ can be the most relevant. These processes also give rise to $\mathbb{E}_{T}$ when the decay products of $f$ include neutrinos. For our analysis we therefore include only

- $\gamma \gamma \rightarrow \tau^{+} \tau^{-}, c \bar{c}$ and $b \bar{b}$

contributions from Isajet using a photon PDF which again includes a beam/bremsstrahlung convolution $[53,80]$.

\section{Detection of higgsinos at a higgsino factory}

\subsection{Benchmark ILC1 at $\sqrt{s}=250 \mathrm{GeV}$}

We begin by first discussing higgsino pair production for the ILC1 benchmark point with $\sqrt{s}=250 \mathrm{GeV}$, the nominal turn-on energy of the ILC. Once threshold for pair production is passed, then the two higgsino pair production reactions occurring at the highest rates are

- $e^{+} e^{-} \rightarrow \widetilde{W}_{1}^{+} \widetilde{W}_{1}^{-} \rightarrow\left(f \bar{f}^{\prime} \widetilde{Z}_{1}\right)+\left(F \bar{F}^{\prime} \widetilde{Z}_{1}\right)$ and

- $e^{+} e^{-} \rightarrow \widetilde{Z}_{1} \widetilde{Z}_{2} \rightarrow \widetilde{Z}_{1}+\left(f \bar{f} \widetilde{Z}_{1}\right)$

where $f$ and $F$ are SM fermions. For models where $|\mu| \ll M_{1,2}$, the two lightest neutralinos are well approximated by $\frac{\left(\tilde{h}_{u} \pm \tilde{h}_{d}\right)}{\sqrt{2}}$, and the coupling of $Z$ to $\widetilde{Z}_{1} \widetilde{Z}_{1}$ and $\widetilde{Z}_{2} \widetilde{Z}_{2}$ pairs is dynamically suppressed [81]. Thus, though the phase space for $\widetilde{Z}_{2} \widetilde{Z}_{2}$ production is qualitatively 


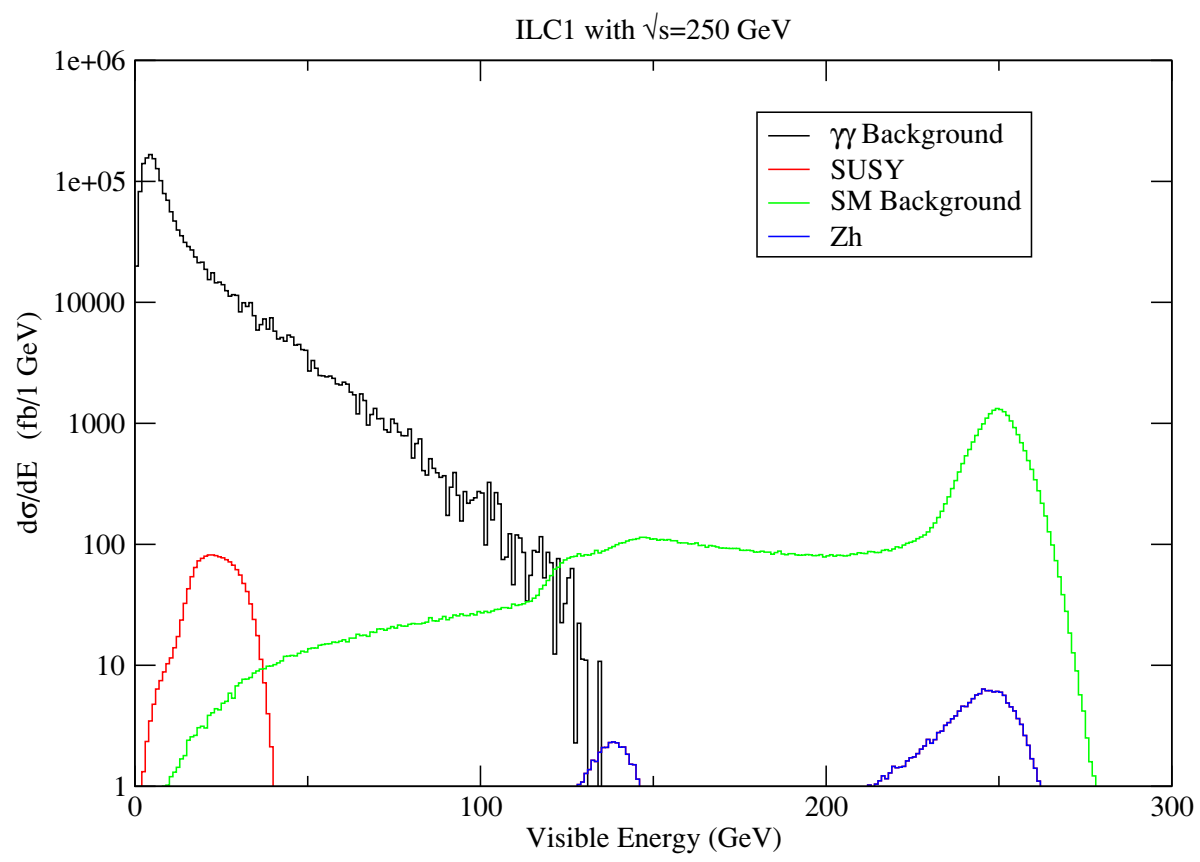

Figure 4. Distribution in visible energy measured in $e^{+} e^{-}$events at $\sqrt{s}=250 \mathrm{GeV}$ for ILC1 signal and SM backgrounds from $e^{+} e^{-}$and $\gamma \gamma$ collisions. We take beamstrahlung parameters $\Upsilon=0.02$ and $\sigma_{z}=0.3 \mathrm{~mm}$.

similar to that for $\widetilde{Z}_{1} \widetilde{Z}_{2}$ production, $\sigma\left(\widetilde{Z}_{2} \widetilde{Z}_{2}\right)$ is much smaller in the RNS framework: see figure 2.

Since $m_{\widetilde{Z}_{1}}$ is only slightly smaller than $m_{\widetilde{W}_{1}, \widetilde{Z}_{2}}$, most of the collision energy ends up in the rest mass $2 m_{\widetilde{Z}_{1}}$ of the LSPs, and the visible final state fermions are relatively soft. To illustrate this, we show in figure 4 the visible energy distribution expected at ILC250 for benchmark ILC1. From the figure, we see the bulk of SM background from $e^{+} e^{-}$ annihilation processes (green curve) in (4.1) peaks around $E_{v i s} \sim 250 \mathrm{GeV}$, with some spillover to higher values due to detector energy mis-measurement. A continuous $E_{v i s}$ tail occurs at lower values due to production of $W W, Z Z, b \bar{b}$ etc. where substantial energy is lost due to decays to neutrinos. There are also two small bumps at $E_{v i s} \sim 140 \mathrm{GeV}$ and $250 \mathrm{GeV}$ arising from $Z h$ production (blue curves). The $140 \mathrm{GeV}$ bump occurs due to $e^{+} e^{-} \rightarrow Z h \rightarrow(\nu \bar{\nu})+(b \bar{b})$. The SUSY signal distribution is depicted by the bounded (red) histogram with $E_{\text {vis }} \sim 0-40 \mathrm{GeV}$ is already well-separated from the $2 \rightarrow 2 \mathrm{SM}$ backgrounds. However, as anticipated, backgrounds from the $2 \rightarrow 4$ processes, $\gamma \gamma \rightarrow c \bar{c}, b \bar{b}$ and $\tau \bar{\tau}$, shown as the black histogram overwhelm the signal by a factor of $\sim 100-1000$.

To select out signal events, we first require:

- $20 \mathrm{GeV}<E_{v i s}<50 \mathrm{GeV}$.

The $\gamma \gamma$ background yields mainly soft visible energy events with a tail extending to higher values. To differentiate signal from this background, we plot in figure 5 the missing transverse energy distribution $d \sigma / d E_{T}$ after the visible energy cut. We see that the $\gamma \gamma$ background falls very rapidly since $E_{T}$ occurs mainly due to neutrinos from the decays of 


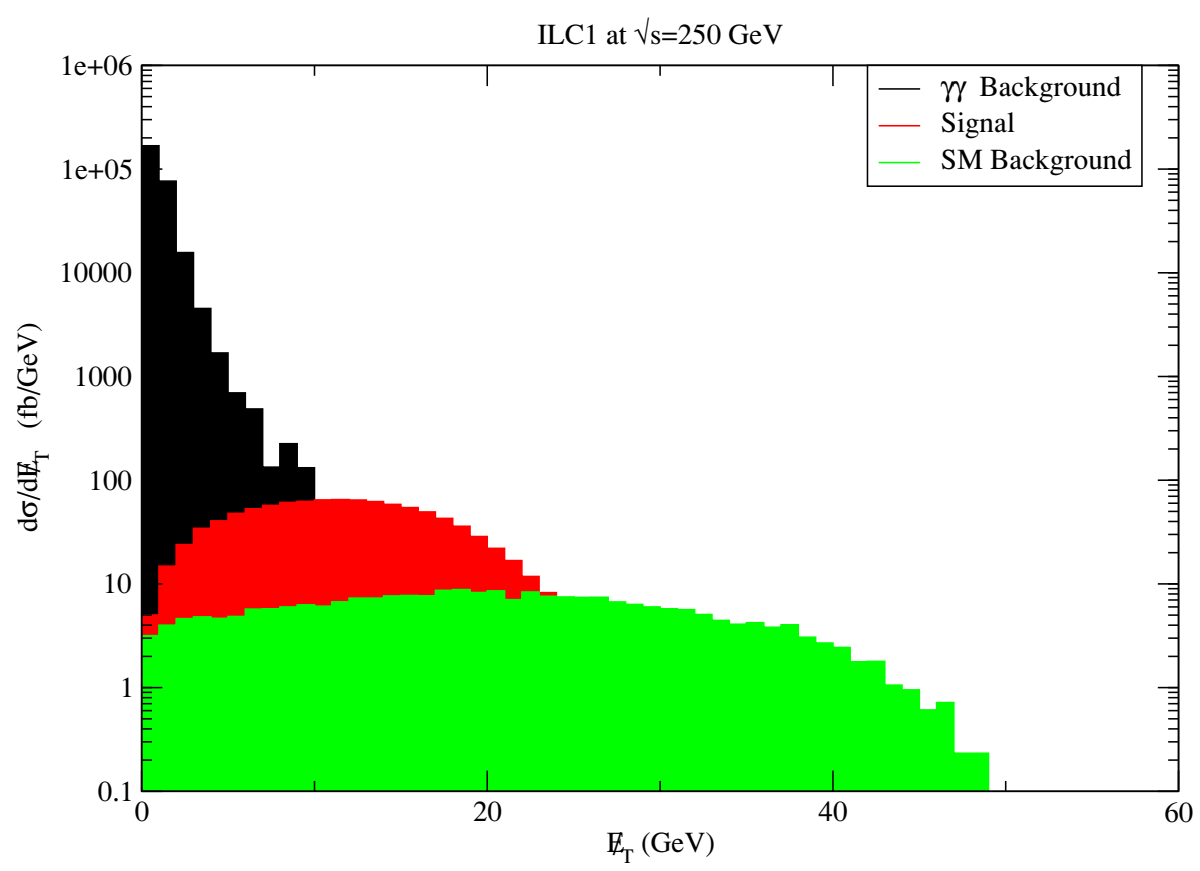

Figure 5. Distribution of missing transverse energy from $e^{+} e^{-}$collisions at $\sqrt{s}=250 \mathrm{GeV}$ for ILC1 signal along with SM background from $e^{+} e^{-}$and $\gamma \gamma$ collisions. We require $20 \mathrm{GeV}<E_{v i s}<50 \mathrm{GeV}$. We take beamstrahlung parameters $\Upsilon=0.02$ and $\sigma_{z}=0.3 \mathrm{~mm}$.

the relatively light $c, b$ and $\tau$, and the signal emerges from $\gamma \gamma$ background if we require $E_{T}>10 \mathrm{GeV}$. The bulge of events with low $E_{v i s}$ but modest $E_{T}$ would herald the discovery of new physics. This also explains why we did not include $\gamma \gamma \rightarrow f \bar{f}$ processes with $f=e, \mu, u, d, s$ in our analysis. These yield back-to-back events in the transverse plane, with essentially no $E_{T}$, and are efficiently eliminated by a $E_{T}$ cut. Thus, for our new physics event sample, we will also require

- $E_{T}>10 \mathrm{GeV}$.

To understand the expected event topologies, we examine the multiplicity of isolated leptons and identied jets. These distributions are shown in figure 6 . We see that the most lucrative signal channels from the perspective of the signal to background ratio appears to be the $n(\ell)=0$ and $n(j e t)=1-3$ bins to which neutralino and chargino production can contribute. To cleanly separate chargino and neutralino contributions so that each particle can be studied in detail, it is also useful to examine other channels.

Before turning to this, we note that the observation of an excess above SM in the multijet plus multi-lepton channels, if interpreted in terms of weak scale SUSY, would suggest the production of charginos and neutralinos. The small energy release in these events would point to a small mass gap between the parent particles and the undetected LSP. In the simplest models with gaugino mass unification, this would indicate the production of higgsino-like states, with $|\mu| \ll m_{1 / 2}$ where $\widetilde{W}_{1}, \widetilde{Z}_{2}$ and $\widetilde{Z}_{1}$ are roughly degenerate, and the bino and winos are substantially heavier. However, it is also possible that such events may arise from wino pair production in models with heavy higgsinos, and a bino only slightly 
lighter than the wino-states. It should, however, be possible to distinguish between these possibilities since, as we mentioned in our discussion of figure 3, with unpolarized beams neutralino production is smaller than $0.1 \mathrm{fb}$ (i.e. three orders of magnitide below expectations for higgsino-like neutralinos) in the latter case. Moreover, the chargino signal from the production of wino-like charginos will reduce much more sharply for right-handed electron beams than for higgsino-like charginos. It should thus be possible to unambiguously conclude that the signal is from higgsino-like, and not gaugino-like super-partners.

With this in mind, we turn to strategies that will help us to obtain essentially pure samples of chargino and of neutralino events for the ILC1 point under examination.

\subsubsection{Chargino pair production}

To select out a nearly pure sample of chargino pair events where the jets all arise from the same chargino, we will first select events with the $E_{v i s}$ and $E_{T}$ cuts introduced above, but also require

$$
\text { - } n(\ell)=1
$$

and

$$
\text { - } n(j e t)=2 \text {. }
$$

After these requirements, we are left with a signal cross section of $6.43 \mathrm{fb}$. Just one background event passes cuts, leading to $\sigma_{B G} \sim 0.018 \mathrm{fb}$, i.e. we have a nearly pure sample of chargino pair events, where one chargino decays leptonically and the other decays hadronically.

We show a scatter plot of these selected events in the $E(j j)$ vs. $m(j j)$ plane in figure 7 . The $m(j j)$ distribution is expected to be bounded from above by $m_{\widetilde{W}_{1}}-m_{\widetilde{Z}_{1}}=14.6 \mathrm{GeV}$ up to energy mis-measurement corrections; this cut-off is seen in figure 7, from which it is apparent the $m_{\widetilde{W}_{1}}-m_{\widetilde{Z}_{1}}$ mass gap is $\sim 15 \mathrm{GeV}$.

The sparticle masses $m_{\widetilde{W}_{1}}$ and $m_{\widetilde{Z}_{1}}$ can be obtained from fits of the $E(j j)$ data distribution [50-52] to various expected theory distributions which vary depending on $m_{\widetilde{W}_{1}}$ and $m_{\widetilde{Z}_{1}}$. The lower endpoint of $E(j j)$ is determined largely by our $E(j)>5 \mathrm{GeV}$ jet requirement but the upper endpoint is quite sensitive to $m_{\widetilde{W}_{1}}$ and $m_{\widetilde{Z}_{1}}$ values.

To assess the precision which can be attained, we generate a synthetic "data" set assuming $100 \mathrm{fb}^{-1}$ of integrated luminosity, along with expected statistical error bars. We also generate theory sample of distributions run over a large grid of $\mu$ and $m_{1 / 2}$ points (which yields a corresponding grid of $m_{\widetilde{W}_{1}}$ and $m_{\widetilde{Z}_{1}}$ points) where each theory sample is run with 10 times the statistics of data. Our analysis ignores any sensitivity to other parameters, and implicitly assumes that we can distinguish between higgsino- and wino-like chargino events (which should be possible as noted just before the start of section 5.1.1). We then compare the $E(j j)$ "data" distribution (with $1 \mathrm{GeV}$ bins) to these theory templates and obtain the values of $\chi^{2}$ between the "data" and the theory. We fix the normalization of theory curves to match "data" so that we are fitting only the shape of the distribution. To obtain the $\chi^{2}$, we add the appropriately weighted statistical errors for the theory and data sets in quadrature. 

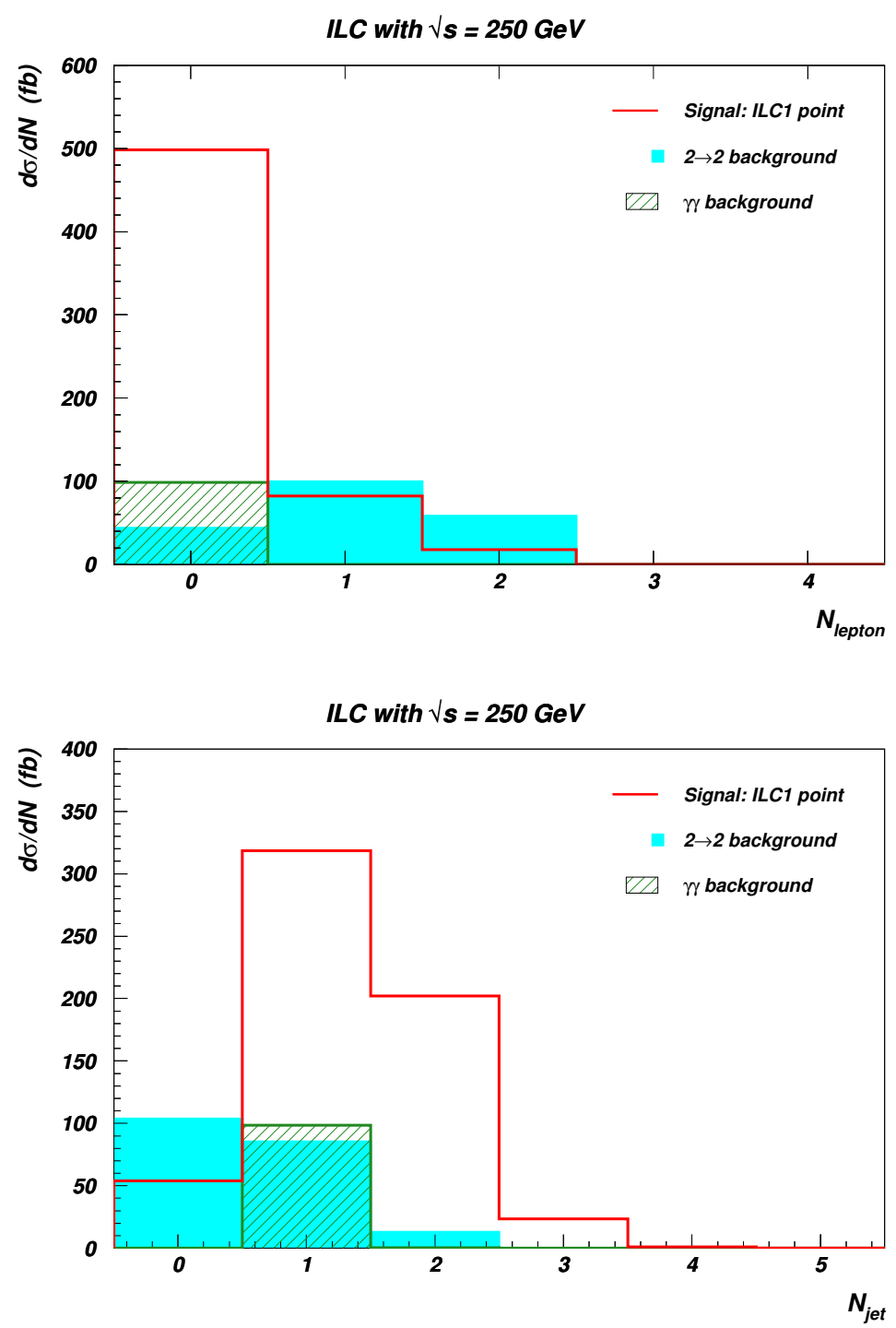

Figure 6. Distribution of $a$ ) isolated lepton multiplicity and $b$ ) jet multiplicity from $e^{+} e^{-}$collisions at $\sqrt{s}=250 \mathrm{GeV}$ for higgsino signals from the ILC1 case study along with corresponding SM backgrounds from $e^{+} e^{-}$and $\gamma \gamma$ collisions. We require $20 \mathrm{GeV}<E_{v i s}<50 \mathrm{GeV}$ and $\mathbb{E}_{T}>10 \mathrm{GeV}$. We take beamstrahlung parameters $\Upsilon=0.02$ and $\sigma_{z}=0.3 \mathrm{~mm}$.

This procedure enables us to obtain a grid of $\Delta \chi^{2}=\chi^{2}-\chi_{\min }^{2}$ values in the $m_{\widetilde{W}_{1}}-m_{\widetilde{Z}_{1}}$ plane. The reader should keep in mind that our theory calculation is also subject to statistical fluctuations that will be reflected in the distribution of $\Delta \chi^{2}$ values. To enable the reader to personally assess the reliability of the computation, we show in figure $8 a$ these $\Delta \chi^{2}$ values binned by $\Delta \chi^{2}<2.3(1 \sigma \mathrm{CL}), \Delta \chi^{2}<4.6(90 \% \mathrm{CL})$ and $\Delta \chi^{2}>4.6$. We also show the corresponding $1 \sigma$ and $90 \%$ CL error ellipses that we obtain as conservative fits to the $\Delta \chi^{2}$ data. ¿From these error ellipses, we find that the $2-3 \%$ mass measurements

- $m_{\widetilde{W}_{1}}=117.8 \pm 2.8 \mathrm{GeV}(1 \sigma)$, 


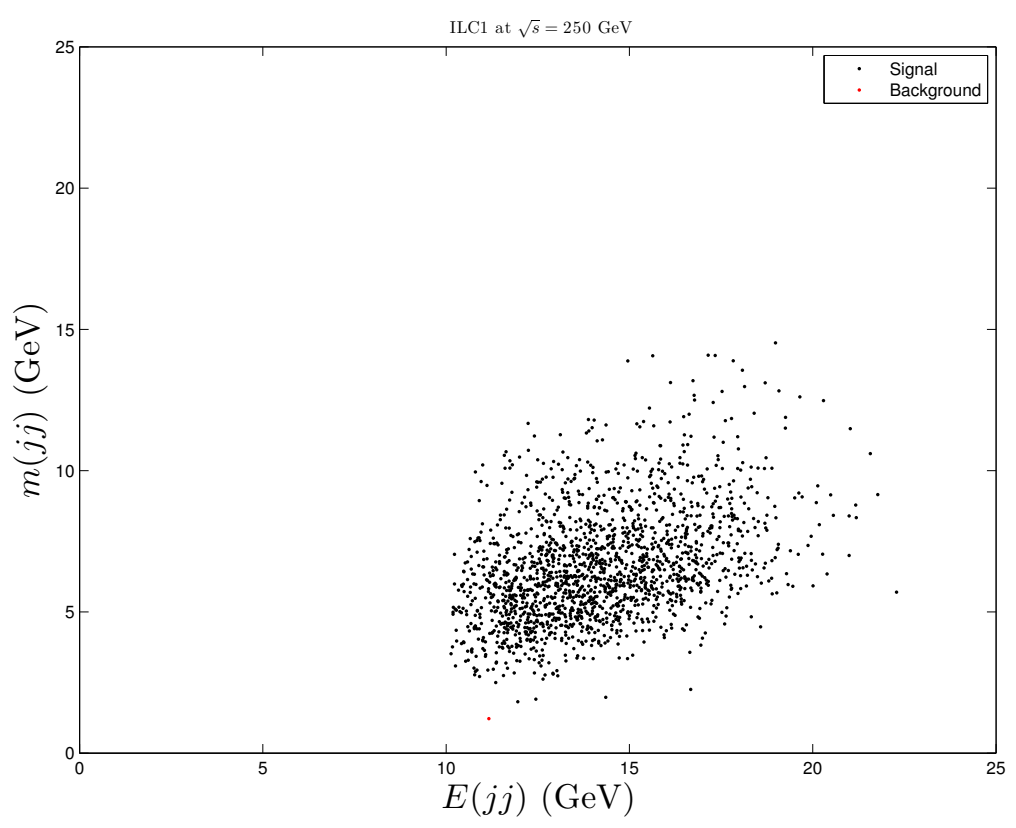

Figure 7. Scatter plot in the $E_{j j}$ vs. $m(j j)$ plane for $1 \ell+2-j e t s$ events from the ILC1 point in $e^{+} e^{-}$collisions at $\sqrt{s}=250 \mathrm{GeV}$. We require $20 \mathrm{GeV}<E_{v i s}<50 \mathrm{GeV}$ and $M E T>10 \mathrm{GeV}$. We take beamstrahlung parameters $\Upsilon=0.02$ and $\sigma_{z}=0.3 \mathrm{~mm}$.

and also,

- $m_{\widetilde{Z}_{1}}=103.1 \pm 2.2 \mathrm{GeV}(1 \sigma)$,

should be possible for the ILC1 point. The synthetic "data", together with statistical error bars corresponding to an integrated luminosity of $100 \mathrm{fb}^{-1}$, are shown in figure $8 b$ ) along with the best fit distribution shown as the solid curve.

Here, we remark that if instead the time is taken to perform various total cross section measurements around the higgsino pair threshold - which will require a much higher integrated luminosity investment at several $\sqrt{s}$ values $[82,83]$ - even better precision on the masses may be expected.

\subsubsection{Neutralino pair production}

For the case of $\widetilde{Z}_{1} \widetilde{Z}_{2}$ pair production, we examined events where $\widetilde{Z}_{2} \rightarrow q \bar{q} \widetilde{Z}_{1}$ that yield an $n(\ell)=0, n(j)=2$ sample as well as events where $\widetilde{Z}_{2} \rightarrow \ell^{+} \ell^{-} \widetilde{Z}_{1}$, for which $n(\ell)=2$ and $n(j)=0$. While one might expect the dijet sample to yield more events due to the large $\widetilde{Z}_{2} \rightarrow \widetilde{Z}_{1} q \bar{q}$ branching fraction, in fact we find after cuts that the $\ell^{+} \ell^{-}$sample is larger. This is because frequently the two possible quark jets merge to yield only a single resolvable jet given our jet finding algorithm, or else one of the possible jets becomes too soft or too forward to be identified.

For the opposite-sign/same flavor (OS/SF) dilepton signal that we focus on, we use a polarized electron beam with $P_{L}\left(e^{-}\right)=-0.9$ since this helps to reduce potential back- 

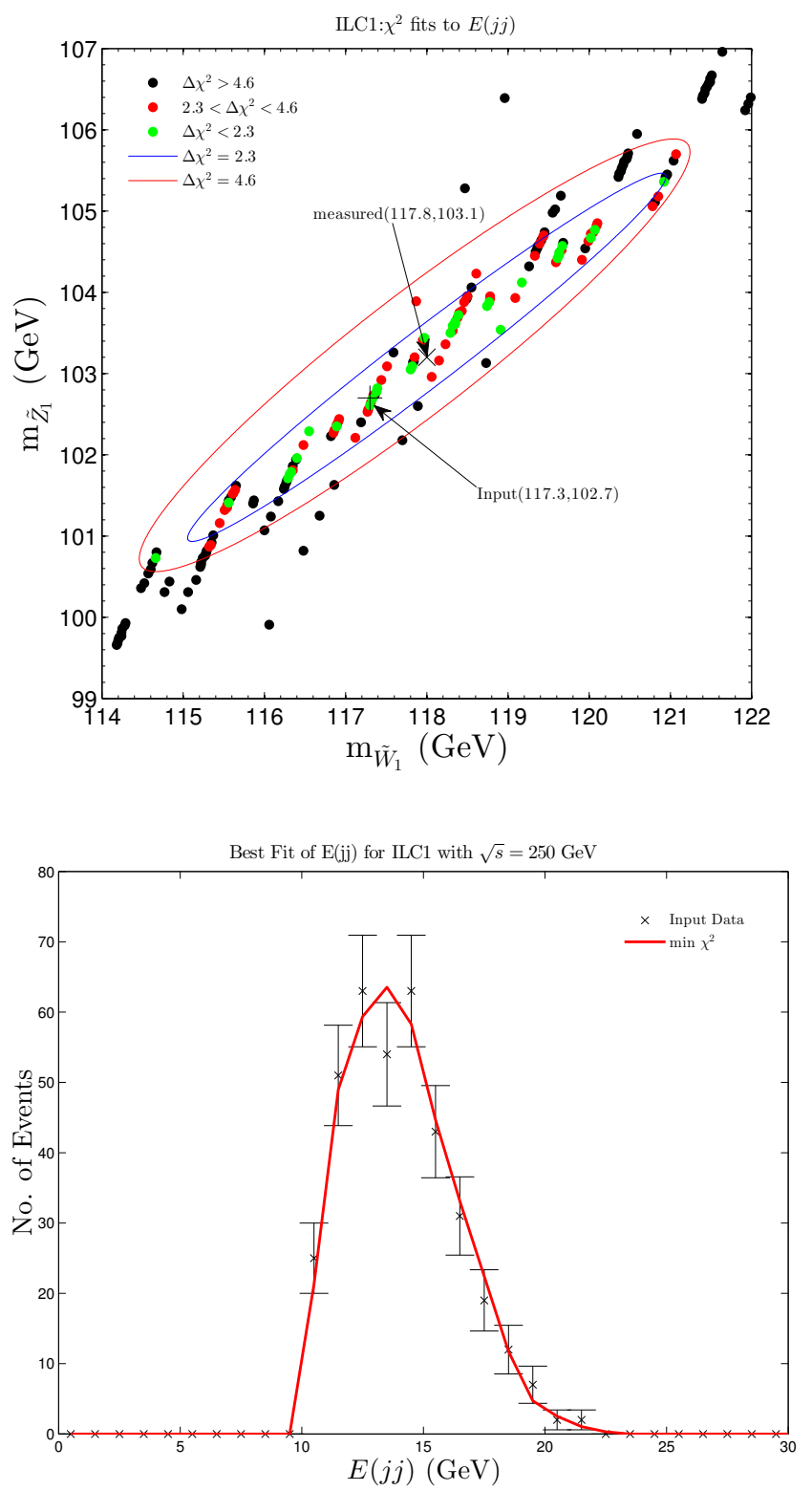

Figure 8. In $a$ ), we show values of $\Delta \chi^{2}$ found from matching $100 \mathrm{fb}^{-1}$ of ILC1 "data" to various "theory" distributions generated from a scan over $\mu$ vs. $m_{1 / 2}$ space. Each "theory" point is run with ten times the events contained in the "data" distribution. We also show fitted error ellipses corresponding to $1 \sigma$ and $90 \%$ CL measurements. In $b)$, we show the distribution in $E(j j)$ from $100 \mathrm{fb}^{-1}$ of "data" along with best fit distribution.

grounds from $W W$ production, and also limits contamination from chargino production to around $10 \%$. We then require:

- exactly 2 OS/SF isolated leptons with no jets,

- $E_{v i s}<35 \mathrm{GeV}$, 


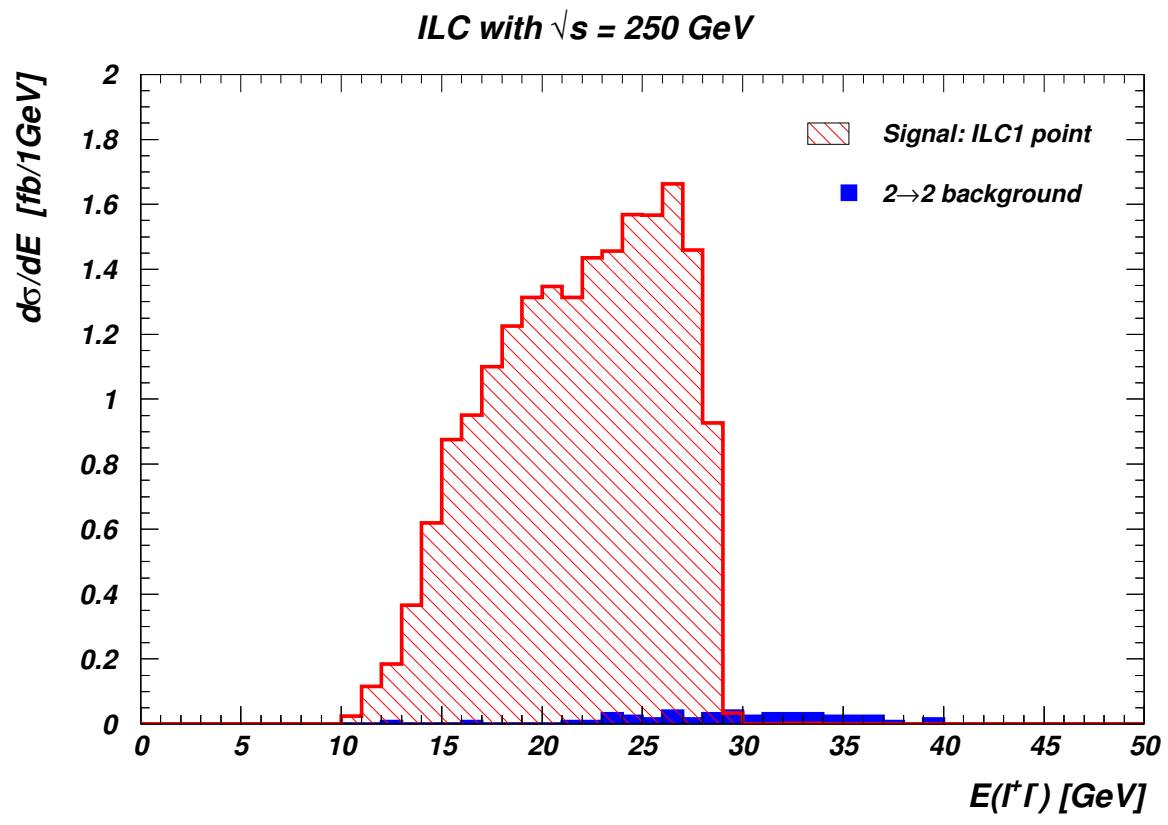

Figure 9. Distribution in $E\left(\ell^{+} \ell^{-}\right)$from $100 \mathrm{fb}^{-1}$ of "data" of OS/SF dilepton events with $P_{L}\left(e^{-}\right)=$ -0.9 from the signal for the ILC1 case, and from SM background (which only comes from $2 \rightarrow 2$ processes), after the $E_{v i s}$ and $\Delta \phi(\ell \ell)$ cuts discussed in the text.

- transverse plane angle between the two leptons $\Delta \phi\left(\ell^{+} \ell^{-}\right)<\pi / 2$.

After these cuts, the $\gamma \gamma$ background is eliminated but some SM background - mainly $W W$ production - remains. The $E\left(\ell^{+} \ell^{-}\right)$distribution after these cuts is shown in figure 9 . This leaves a OS/SF dilepton signal of $19.55 \mathrm{fb}$ while $\mathrm{SM}$ background is $0.44 \mathrm{fb}$. We have checked that the signal has a negligible contribution from chargino production.

Armed with the clean sample of OS/SF dilepton signal events from essentially $\widetilde{Z}_{1} \widetilde{Z}_{2}$ production, we next examine the $m\left(\ell^{+} \ell^{-}\right)$distribution for the ILC1 case. We expect that this distribution is kinematically bounded from above by the $m_{\widetilde{Z}_{2}}-m_{\widetilde{Z}_{1}}$ mass difference and relatively insensitive to the absolute masses of the particles. We use the theory templates generated with 10 times the statistics, as described in section 5.1.1 to obtain a map of $\chi^{2}$ vs. $m_{\widetilde{Z}_{2}}-m_{\widetilde{Z}_{1}}$, shown by the jagged (black) line in figure $10 a$. As before, we fit to the shape, allowing the normalization to float. While statistical fluctuations do contribute to the jaggedness, we have checked that the points with the largest $\chi^{2}$ values come from theory templates where the mass scale of the neutralinos is very different. Also shown in the figure is a parabolic fit to the values of $\chi^{2}$. We see that the mass gap should be measureable at the percent level:

- $m_{\widetilde{Z}_{2}}-m_{\widetilde{Z}_{1}}=21.0 \pm 0.2 \mathrm{GeV}(1 \sigma)$.

The best fit line and the dilepton mass "data" used to obtain the fit are shown in the lower frame in the figure. We see that the shape of this mass distribution is indicative 

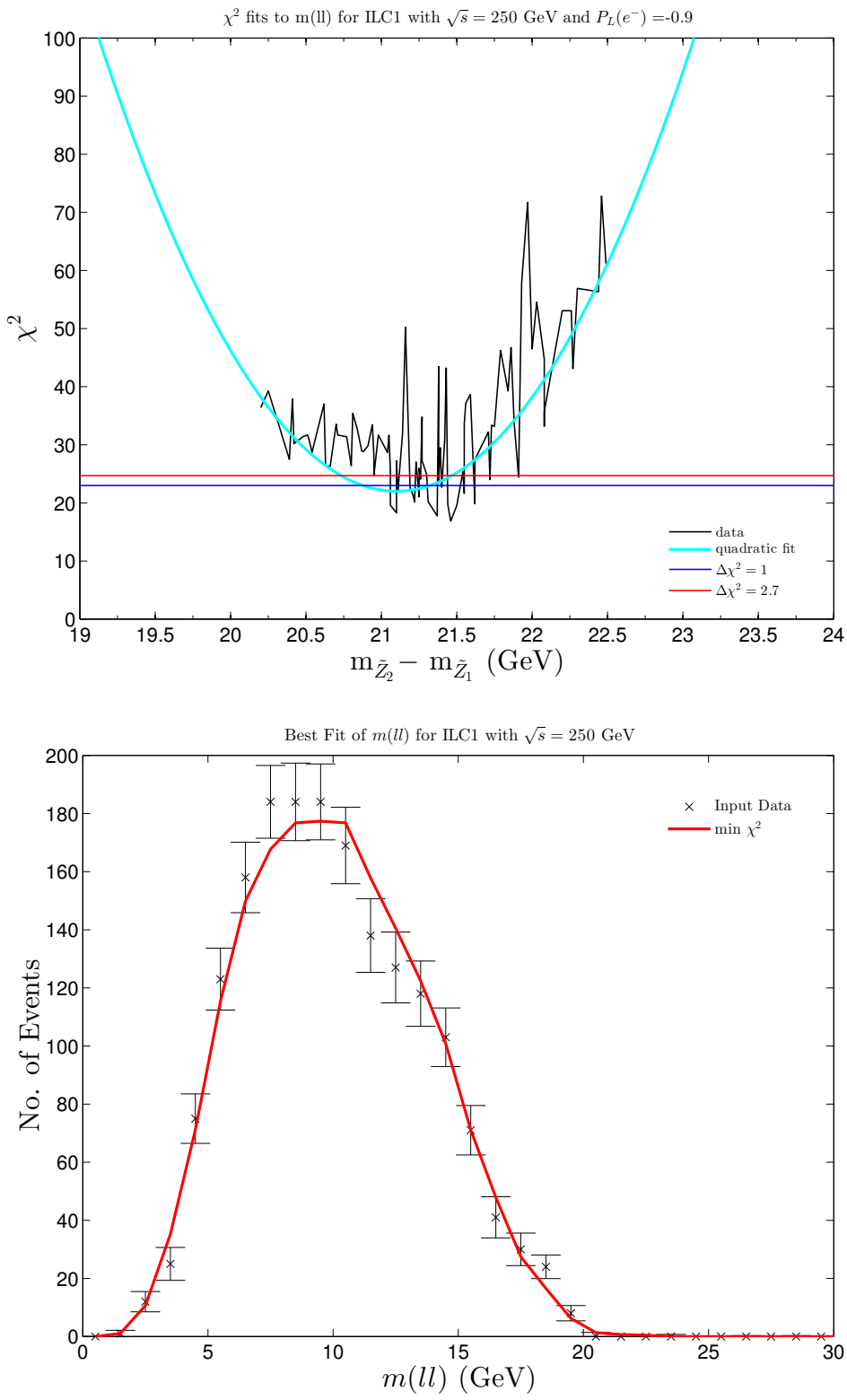

Figure 10. In $a)$, we show values of $\chi^{2}$ vs. $m\left(\ell^{+} \ell^{-}\right)$from $100 \mathrm{fb}^{-1}$ of OS/SF dilepton ILC1 "data" from $\widetilde{Z}_{1} \widetilde{Z}_{2}$ production fit to the shapes from various "theory" templates, as described in the text. In $b$ ), we show the ILC1 "data" for the $m\left(\ell^{+} \ell^{-}\right)$distribution from $\widetilde{Z}_{1} \widetilde{Z}_{2}$ events along with statistical error for $100 \mathrm{fb}^{-1}$. The solid curve shows the best fit to these "data".

of an opposite sign of the $\widetilde{Z}_{1}$ and $\widetilde{Z}_{2}$ mass eigenvalues [84], completely compatible with expectation [85] from the decay of a higgsino-like $\widetilde{Z}_{2}$ to a higgsino-like $\widetilde{Z}_{1}$.

Once the mass gap is known, it is possible to extract the neutralino mass value via a fit to the $E\left(\ell^{+} \ell^{-}\right)$distribution because the energy of the daughter leptons (but not their invariant mass) depends on the boost of the parent $\widetilde{Z}_{2}$. We use the same procedure described above to fit the $100 \mathrm{fb}^{-1} \mathrm{OS} / \mathrm{SF}$ dilepton $E\left(\ell^{+} \ell^{-}\right)$using theory templates with 
different values of $m_{\widetilde{Z}_{2}}$, but with $m_{\widetilde{Z}_{2}}-m_{\widetilde{Z}_{1}}$ fixed at $21 \mathrm{GeV}$. The corresponding values of $\chi^{2}$ along with the parabolic fit is shown in figure $11 a$. We find that $m_{\widetilde{Z}_{2}}$ is measured as

- $m_{\widetilde{Z}_{2}}=123.7 \pm 0.2 \mathrm{GeV}(1 \sigma)$,

a $0.2 \%$ measurement. Combining the $m_{\widetilde{Z}_{2}}-m_{\widetilde{Z}_{1}}$ and $m_{\widetilde{Z}_{2}}$ measurements also gives $m_{\widetilde{Z}_{1}}$ :

- $m_{\widetilde{Z}_{1}}=102.7 \pm 0.3 \mathrm{GeV}$ (ILC1-dileptons).

This value serves as a consistency check against the measurement of $m_{\widetilde{Z}_{1}}$ from chargino pair production, and most importantly, lends support to the SUSY interpretation of these events. The distribution of $E\left(\ell^{+} \ell^{-}\right)$data are shown in figure $\left.11 b\right)$ along with the corresponding best fit depicted by the solid curve.

\subsection{Benchmark ILC2 at $\sqrt{s}=340 \mathrm{GeV}$}

Benchmark point ILC2 is more challenging for ILC studies because the mass gap between $\widetilde{W}_{1} / \widetilde{Z}_{2}$ and the $\widetilde{Z}_{1}$ is just about $10 \mathrm{GeV}$, resulting typically in softer energy release from three-body $\widetilde{W}_{1}$ and $\widetilde{Z}_{2}$ decays. This mass gap is close to the minimum for RNS models where $\Delta_{E W}^{-1}>3 \%$. Of course, since charginos and neutralinos are heavier, its exploration requires a higher $\sqrt{s}$ to reach higgsino pair production threshold. In this case, we perform studies at $\sqrt{s}=340 \mathrm{GeV}$, enough for $\widetilde{W}_{1}^{+} \widetilde{W}_{1}^{-}$and $\widetilde{Z}_{1} \widetilde{Z}_{2}$ production, but just below $t \bar{t}$ threshold. For these higher $\sqrt{s}$ values, the expected beamstrahlung parameter $\Upsilon$ is expected to increase to 0.03 , while $\sigma_{z}$ remains at $0.3 \mathrm{~mm}$ [79].

The $E_{\text {vis }}$ distribution from signal and background is shown in figure 12 . Here, we see the ILC2 signal restricted to the region with $E_{v i s} \lesssim 30 \mathrm{GeV}$, while the background from $\gamma \gamma$ collisions is more severe than for the $\sqrt{s}=250 \mathrm{GeV}$ case. We impose a cut of

- $E_{\text {vis }}<30 \mathrm{GeV}$.

Following our earlier analysis, we examine the $E_{T}$ in figure 13 . We see that, unlike the case of ILC1, the signal never emerges from the $\gamma \gamma$ background. Clearly additional cuts are necessary for observability of the signal.

\subsubsection{Chargino pair production for ILC2}

To extract a chargino pair production signal from the SM background for benchmark ILC2, we thus require:

- $E_{\text {vis }}<30 \mathrm{GeV}$,

- $E_{T}>10 \mathrm{GeV}$,

- exactly one isolated lepton with $E>5 \mathrm{GeV}$ and one jet with $E(j)>5 \mathrm{GeV}$.

For the case of ILC2, the hadronic energy release from $\widetilde{W}_{1} \rightarrow q \bar{q}^{\prime} \widetilde{Z}_{1}$ is so small that we almost never produce two resolvable jets, making chargino mass extraction difficult via continuum production (although perhaps still possible via threshold scans with sufficient integrated luminosity). Hence, instead we focus on the $n(\ell)=1, n(j e t)=1$ signal. After 

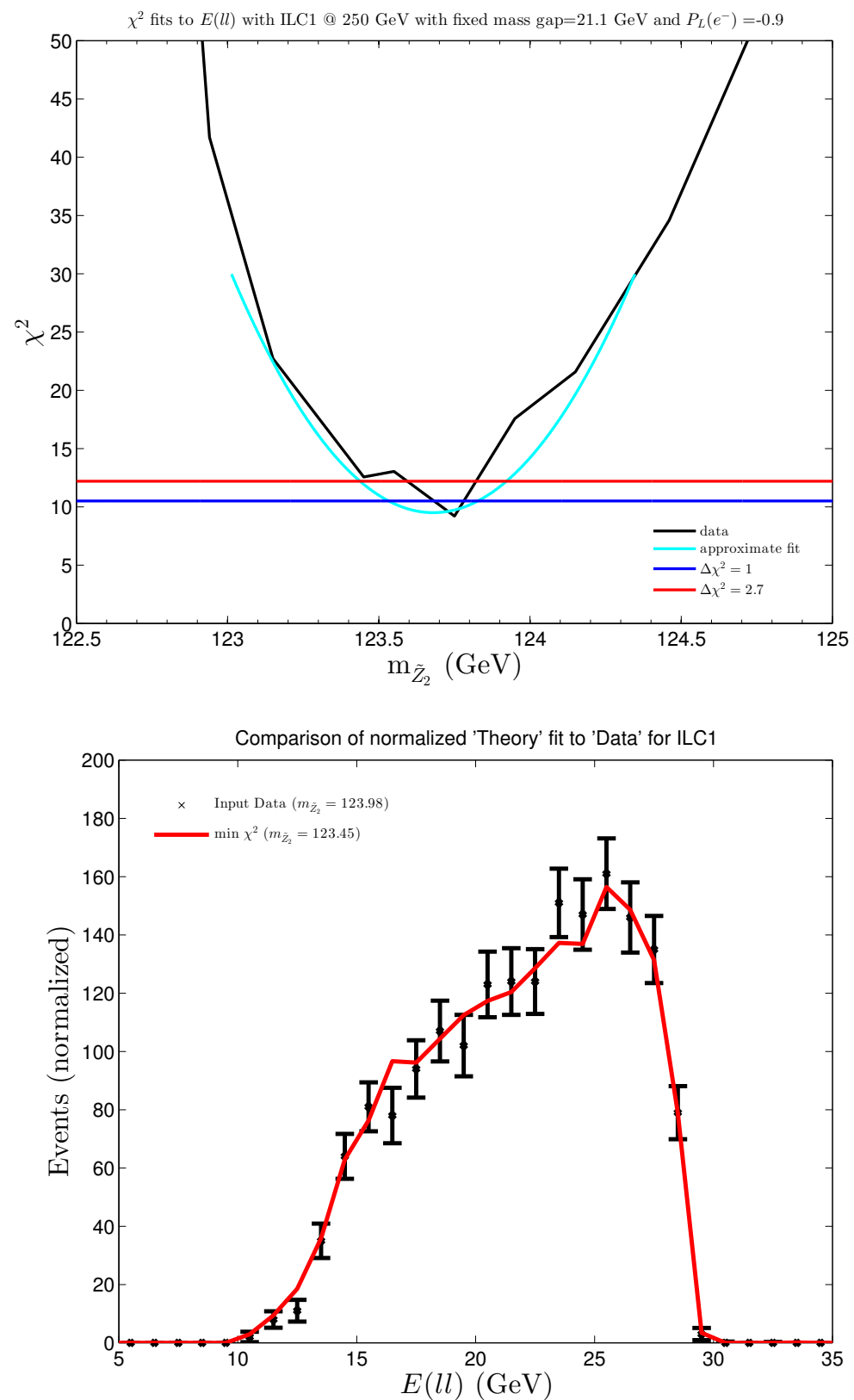

Figure 11. In $a$ ), we show fitted values of $\chi^{2}$ found from matching $100 \mathrm{fb}^{-1}$ of OS/SF dilepton "data" from $\widetilde{Z}_{1} \widetilde{Z}_{2}$ production to various "theory" distributions generated from varying $m_{\widetilde{Z}_{2}}$ while keeping $m_{\widetilde{Z}_{2}}-m_{\widetilde{Z}_{1}}$ fixed at $21 \mathrm{GeV}$. In $\left.b\right)$, we show the distribution in $E\left(\ell^{+} \ell^{-}\right)$from a $100 \mathrm{fb}^{-1}$ of OS/SF dilepton ILC1 "data" from $\widetilde{Z}_{1} \widetilde{Z}_{2}$ production along with best fit.

these requirements, we plot the transverse plane lepton-jet opening angle which is shown in figure 14. Most of the SM background comes from $\gamma \gamma \rightarrow \tau^{+} \tau^{-}$followed by one leptonic and one hadronic tau decay. This may be mostly eliminated by requiring

- $\Delta \phi(\ell, j e t)<120^{\circ}$. 


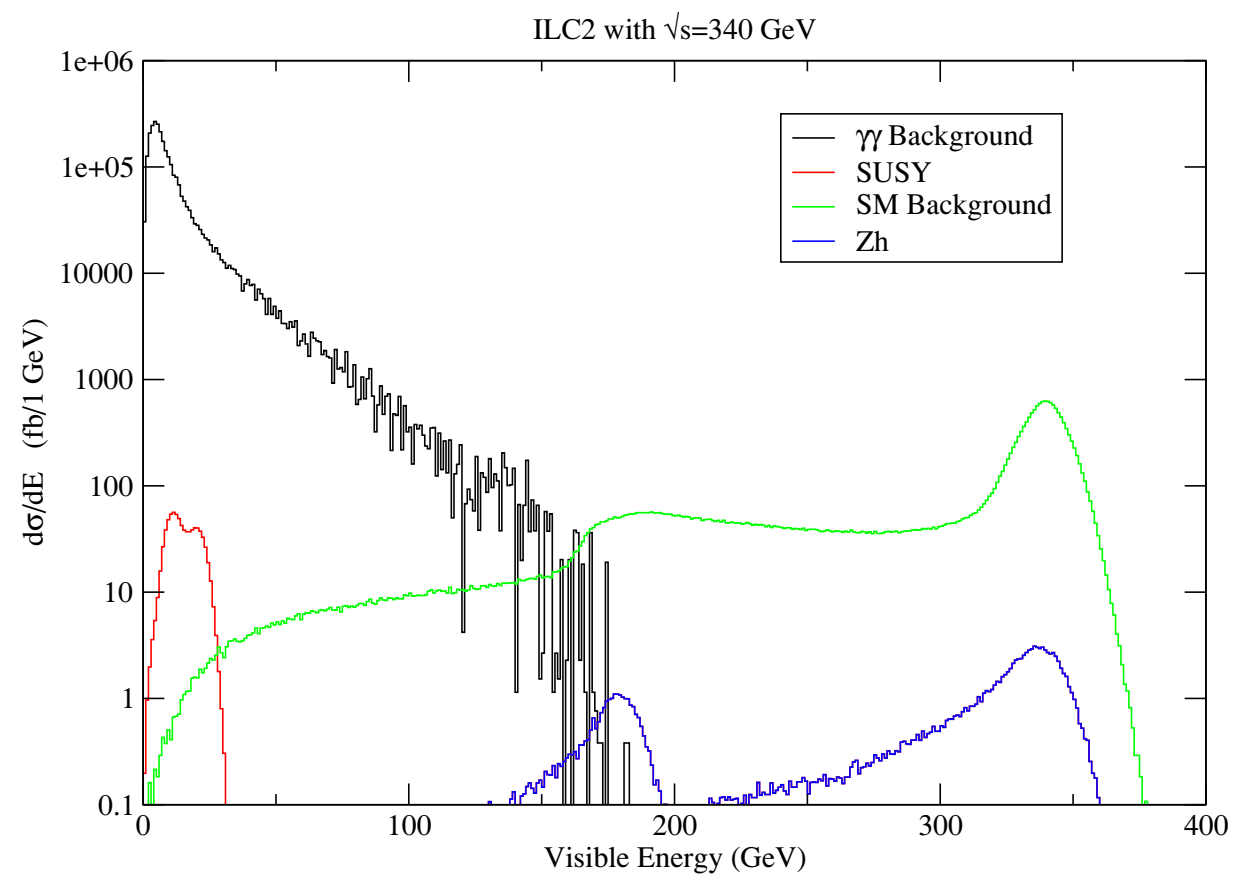

Figure 12. Distribution in $E_{v i s}$ from benchmark ILC2 signal and SM backgrounds at ILC with $\sqrt{s}=340 \mathrm{GeV}$ and $\Upsilon=0.03$.

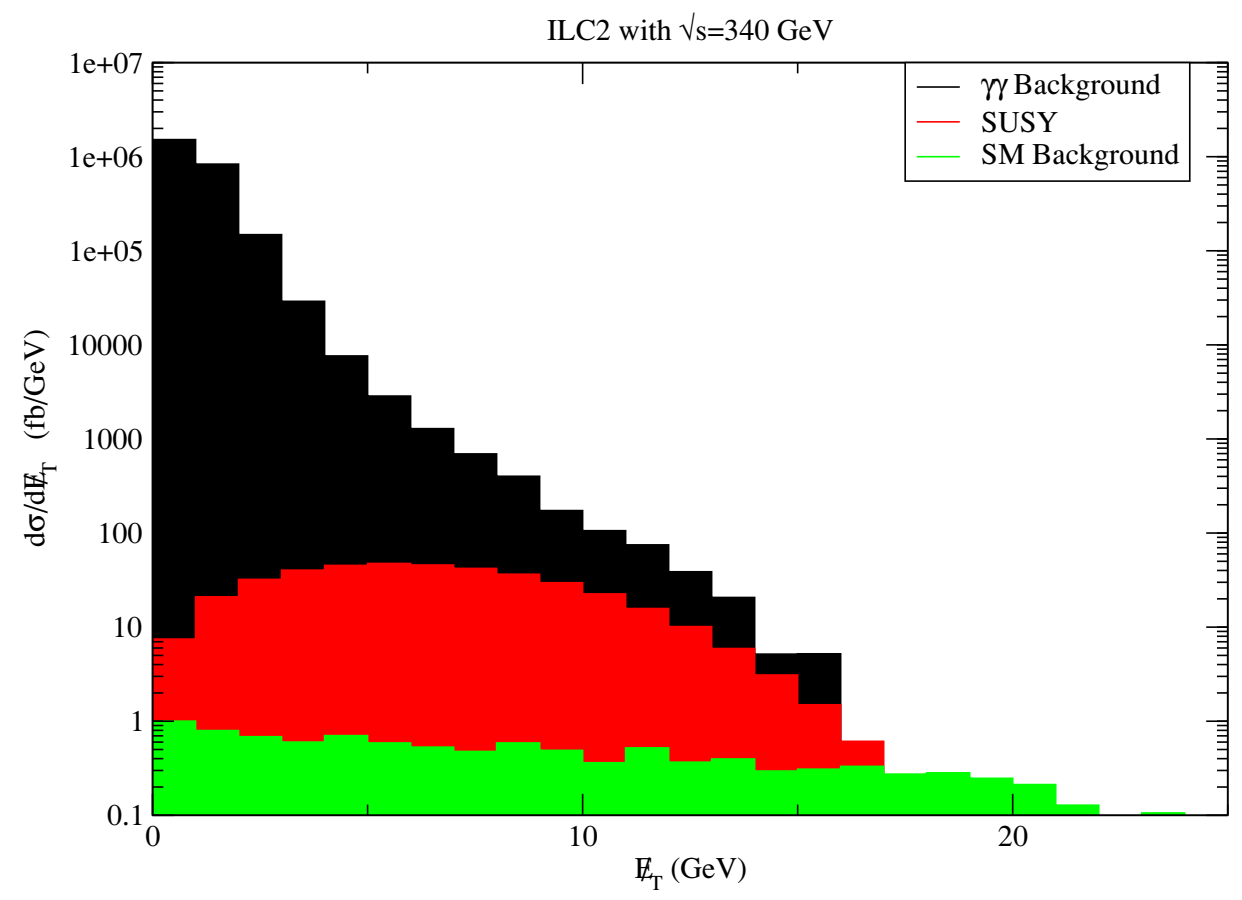

Figure 13. Distribution in missing transverse energy from $e^{+} e^{-}$collisions at $\sqrt{s}=340 \mathrm{GeV}$ for signal from the ILC2 benchmark case, along with SM backgrounds from $e^{+} e^{-}$and $\gamma \gamma$ collisions. We require $E_{v i s}<30 \mathrm{GeV}$. We take beamstrahlung parameters $\Upsilon=0.03$ and $\sigma_{z}=0.3 \mathrm{~mm}$. 


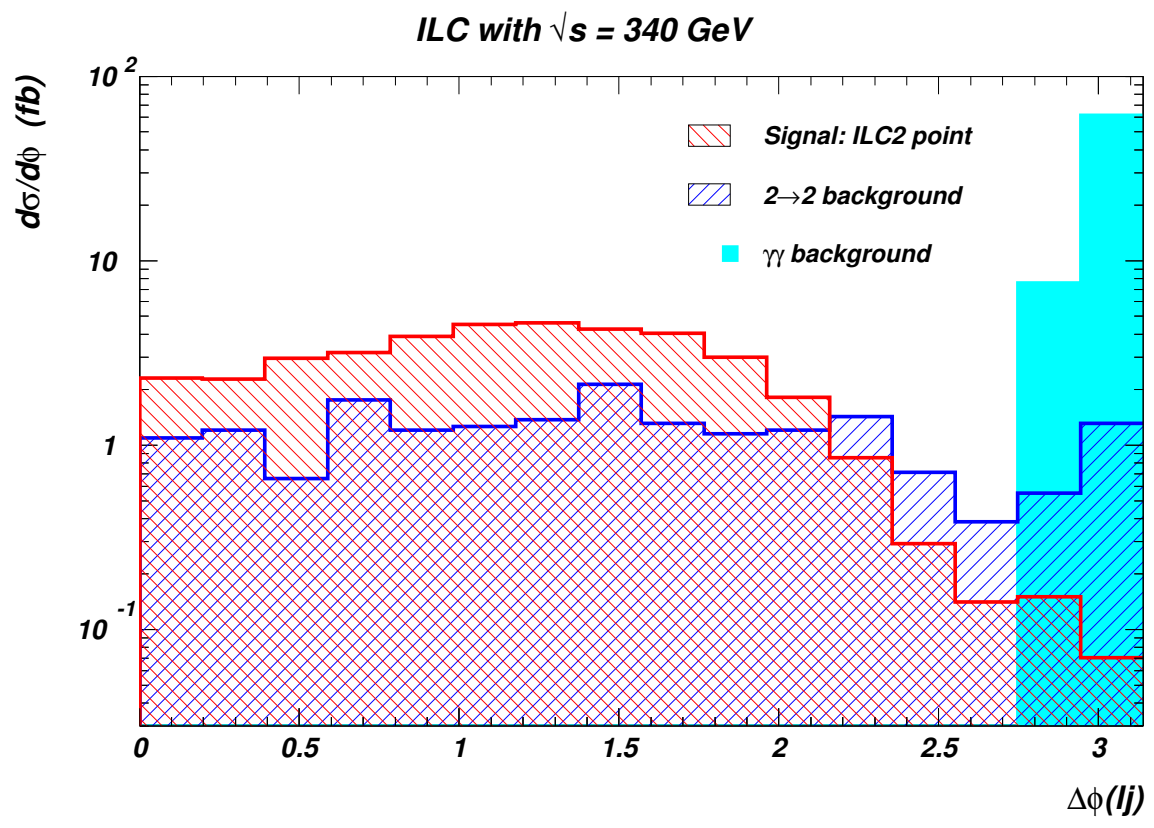

Figure 14. Distribution in transverse plane opening angle between the isolated lepton and jet for the ILC2 signal point at $\sqrt{s}=340 \mathrm{GeV}$, and for SM backgrounds. We require $E_{v i s}<30 \mathrm{GeV}$ and $E_{T}>10 \mathrm{GeV}$. We take beamstrahlung parameters $\Upsilon=0.03$ and $\sigma_{z}=0.3 \mathrm{~mm}$.

After this cut we are left with a signal of $7.1 \mathrm{fb}$ whilst SM background is at the $2.8 \mathrm{fb}$ level, and that all the $\gamma \gamma$ background, which arises from tau pair production, is eliminated. This should not be surprising because most taus that decay into $5 \mathrm{GeV}$ jets/leptons will be significantly boosted, and hence tend to have their decay products nearly back-to-back in the transverse plane. We see that a discovery of new physics might be possible with a data set of just a few $\mathrm{fb}^{-1}$ at ILC340 even in this difficult case.

\subsubsection{Neutralino pair production for ILC2}

As mentioned earlier, if any signal in the $1 \ell 1 j$ channel just discussed is to be attributed to higgsino-like charginos of SUSY, we should also expect a signal from $\widetilde{Z}_{1} \widetilde{Z}_{2}$ production as this reaction must have a comparable production cross section. We are thus led to examine $\widetilde{Z}_{1} \widetilde{Z}_{2}$ production for the ILC2 point with $\sqrt{s}=340 \mathrm{GeV}$, where $\widetilde{Z}_{2} \rightarrow \ell^{+} \ell^{-} \widetilde{Z}_{1}$. This acoplanar dilepton signal may also allow for neutralino mass reconstruction via continuum production. We require

- $E_{v i s}<30 \mathrm{GeV}$,

- a pair of OS/SF leptons, with $n(j)=0$,

- $E_{T}>5 \mathrm{GeV}$.

For this channel, we operate with mainly right polarized electron beams with $P_{L}\left(e^{-}\right)=$ -0.9 to reduce backgrounds from $W^{+} W^{-}$and contamination from $\widetilde{W}_{1}^{+} \widetilde{W}_{1}^{-}$production. 


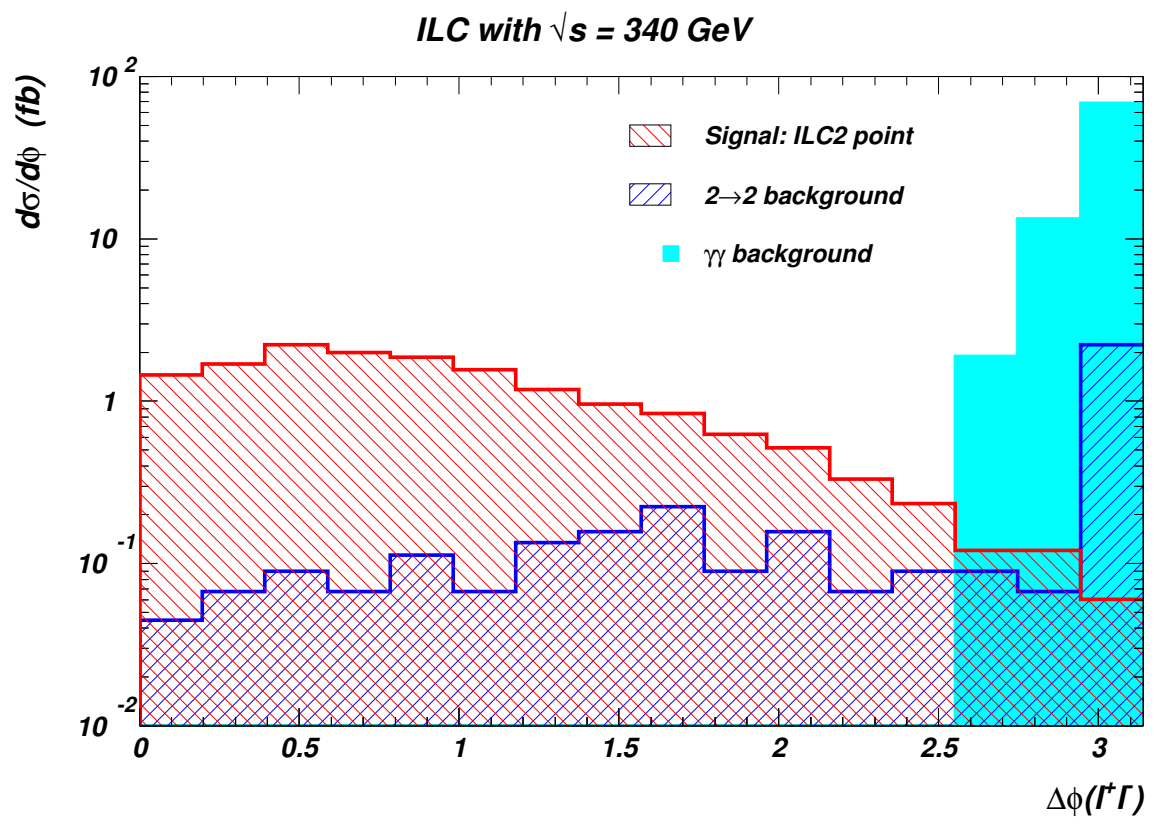

Figure 15. Distribution in transverse opening angle between isolated OS/SF leptons for ILC2 signal at $\sqrt{s}=340 \mathrm{GeV}$ and for SM backgrounds. We require $E_{v i s}<30 \mathrm{GeV}$ and $E_{T}>5 \mathrm{GeV}$. We take beamstrahlung parameters $\Upsilon=0.03$ and $\sigma_{z}=0.3 \mathrm{~mm}$.

We next plot the transverse OS/SF dilepton opening angle in figure 15. To eliminate the $\gamma \gamma$ background which is once again nearly back-to-back in the transverse plane, and to improve the signal-to-background ratio, we also require:

- $\Delta \phi\left(\ell^{+} \ell^{-}\right)<90^{\circ}$.

At this point, we have a signal sample of $2.6 \mathrm{fb}$ while SM background is at the $0.15 \mathrm{fb}$ level with no $\gamma \gamma$ background. Once again, discovery of new physics is possible with just a few $\mathrm{fb}^{-1}$ of integrated luminosity.

For benchmark ILC2, we use the same procedure that we used for the ILC1 case study to extract the neutralino masses. We first fit the normalized theory templates (generated with $1000 \mathrm{fb}^{-1}$ each) with varying $m_{\widetilde{Z}_{2}}-m_{\widetilde{Z}_{1}}$ mass gaps to a $100 \mathrm{fb}^{-1}$ "data" distribution. The various $\chi^{2}$ values along with a parabolic fit are shown in figure $16 a$. As before, we have checked that the very large $\chi^{2}$ values for a mass gap near the bottom of the parabola arise from extreme values of $m_{\widetilde{Z}_{2}}$ in the templates. For the ILC2 case, we find the $m_{\widetilde{Z}_{2}}-m_{\widetilde{Z}_{1}}$ mass gap is measured to be

- $m_{\widetilde{Z}_{2}}-m_{\widetilde{Z}_{1}}=9.7 \pm 0.2 \mathrm{GeV}(1 \sigma)$

a $2 \%$ measurement. The data along with best theory fit are shown in figure $16 b$.

Next, keeping the mass gap fixed near $9.7 \mathrm{GeV}$, we generate theory templates for the $E\left(\ell^{+} \ell^{-}\right)$distributions from $\widetilde{Z}_{1} \widetilde{Z}_{2}$ production with 10 times the statistics of "data" but with varying $m_{\widetilde{Z}_{2}}$ values and fit the shapes of these to the corresponding "data" distribution 

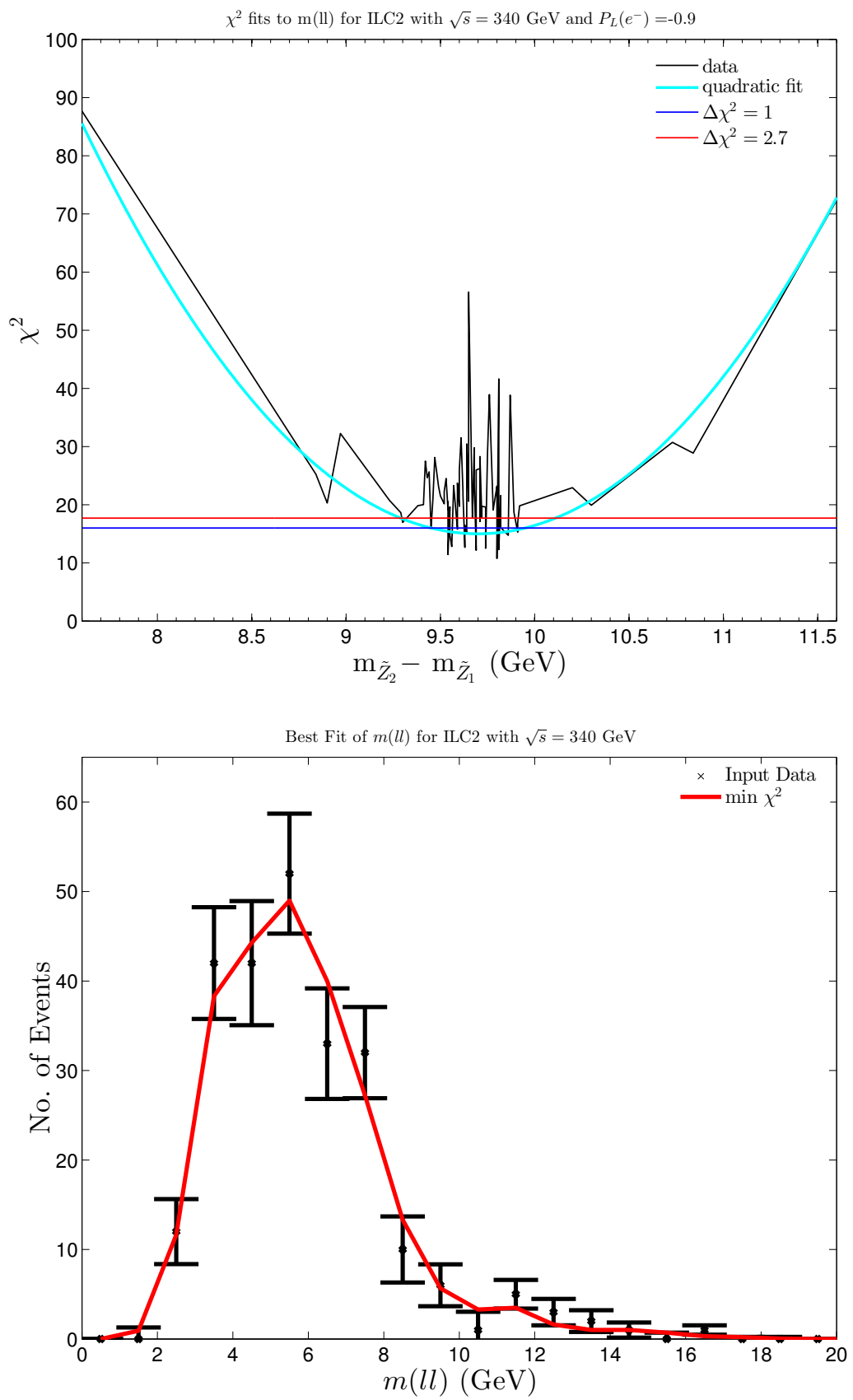

Figure 16. In $a$ ), we show $\chi^{2}$ values vs. $m\left(\ell^{+} \ell^{-}\right)$from $100 \mathrm{fb}^{-1}$ of OS/SF dilepton ILC2 "data" from $\widetilde{Z}_{1} \widetilde{Z}_{2}$ production fit to theory along with a best-fit parabola. In $b$ ), we show the distribution in $m\left(\ell^{+} \ell^{-}\right)$from a $100 \mathrm{fb}^{-1}$ of OS/SF dilepton ILC2 "data" from $\widetilde{Z}_{1} \widetilde{Z}_{2}$ production along with best fit.

as before. In figure 17, we show the $\chi^{2}$ values along with the parabolic fit. We find a measurement of

- $m_{\widetilde{Z}_{2}}=158.5 \pm 0.4 \mathrm{GeV}(1 \sigma)$.

The $E\left(\ell^{+} \ell^{-}\right)$distribution for "data" along with best fit theory are shown in figure $17 b$. By 

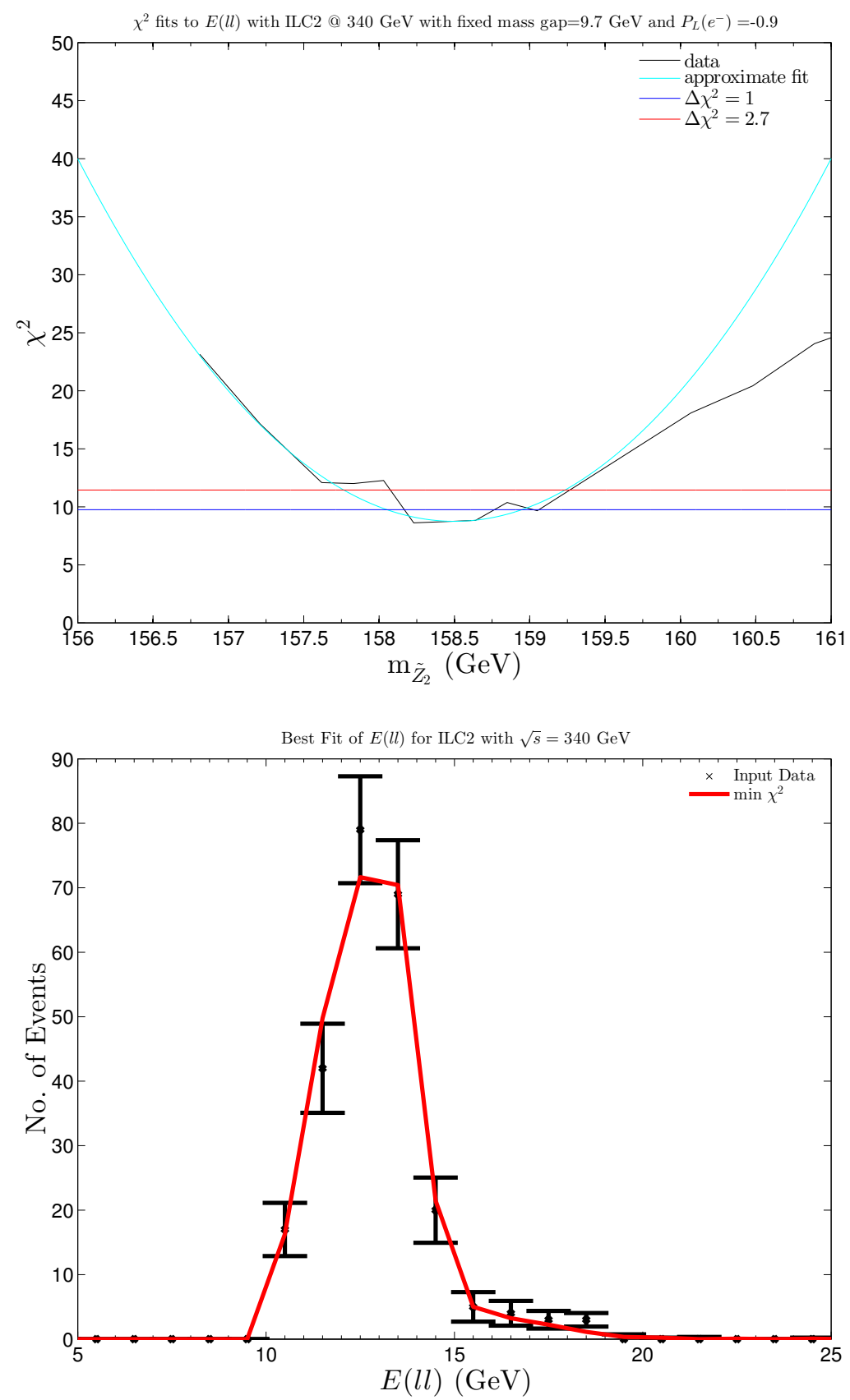

Figure 17. In $a$ ), we show values of $\chi^{2}$ found from matching $100 \mathrm{fb}^{-1}$ of OS/SF dilepton ILC2 "data" from $\widetilde{Z}_{1} \widetilde{Z}_{2}$ production to various "theory" distributions generated from varying $m_{\widetilde{Z}_{2}}$ while keeping $m_{\widetilde{Z}_{2}}-m_{\widetilde{Z}_{1}}$ fixed at $9.7 \mathrm{GeV}$. In $\left.b\right)$, we show the distribution in $E\left(\ell^{+} \ell^{-}\right)$from a $100 \mathrm{fb}^{-1}$ of OS/SF dilepton ILC2 "data" from $\widetilde{Z}_{1} \widetilde{Z}_{2}$ production along with best fit.

combining the $m_{\widetilde{Z}_{2}}$ and mass gap measurements, we find

- $m_{\widetilde{Z}_{1}}=148.8 \pm 0.5 \mathrm{GeV}$ (ILC2-dileptons). 


\section{Conclusions}

Naturalness arguments imply small $|\mu|$, and concomitantly four light higgsino-like states with masses $\sim 100-300 \mathrm{GeV}$, the closer to $M_{Z}$ the more natural. Because of the small energy release in their decays, direct higgsino production may be difficult-to-impossible to detect at LHC14, while the International Linear $e^{+} e^{-}$Collider would be a higgsino factory and serve as a SUSY discovery machine so long as $\sqrt{s}>2 m$ (higgsino). We investigated two benchmark scenarios: ILC1 with lighter higgsinos $\sim 120 \mathrm{GeV}$ and mass gap $\sim 15-22 \mathrm{GeV}$ relative to the LSP, and ILC2 with heavier higgsinos $\sim 150 \mathrm{GeV}$ but with a mass gap of just $10 \mathrm{GeV}$, close to the minimum possible in models with no worse than $3 \%$ fine-tuning.

For both these cases, the chargino pair and neutralino pair signals should be seen above usual SM $2 \rightarrow 2$ background and $\gamma \gamma$ induced events via a combination of specially devised $E_{v i s}, E_{T}$, angle and topology cuts with an integrated luminosity of just a few fb ${ }^{-1}$. The signal will be characterized by low $E_{v i s}(\lesssim 30-50 \mathrm{GeV})$ plus $E_{T}$ events indicating the production of heavy parents that decay into an invisible partner with a mass just 10$20 \mathrm{GeV}$ lighter. Observation of a signal in both $j e t(s)+\ell$ and OS/SF dilepton channels at the expected rates will point to the production of higgsinos that are the hallmark of natural SUSY models. For ILC1, the $\ell+j$ jets signal allows for a continuum measurement of $m_{\widetilde{W}_{1}}$ and $m_{\widetilde{Z}_{1}}$ to $\sim 2 \%$ accuracy assuming a canonical value of $100 \mathrm{fb}^{-1}$ of integrated luminosity. The neutralino pair production reaction can be seen above the background in the OS/SF, same-side dilepton signal which allows for the mass gap $m_{\widetilde{Z}_{2}}-m_{\widetilde{Z}_{1}}$ to be measured via the $m\left(\ell^{+} \ell^{-}\right)$distribution to $\sim 1 \%$ accuracy while $m_{\widetilde{Z}_{2}}$ can be measured to sub-GeV precision.

The more challenging ILC2 point allows for chargino pairs to be seen above background, but the smaller $m_{\widetilde{W}_{1}}-m_{\widetilde{Z}_{1}}$ mass gap makes dijet resolution very difficult so that a continuum mass measurement via $E(j j)$ is not possible using our simple methods. The OS/SF same-side dilepton measurement still remains viable in the case of ILC2 where the mass gap $m_{\widetilde{Z}_{2}}-m_{\widetilde{Z}_{1}}$ can be measured to $\sim 2 \%$ accuracy and $m_{\widetilde{Z}_{2}}$ can be again measured to sub-GeV precision.

Although we have performed our analysis using the RNS model as a guide, our results should be applicable to all models with light higgsinos. We are encouraged that even the difficult point with the smallest mass gap for $150 \mathrm{GeV}$ higgsinos allows not only detection, but also precision mass measurements, even at a centre-of-mass energy just modestly above the production threshold. This leads us to infer that an electron-positron collider will serve as a definitive probe of the idea of naturalness in all SUSY models where the superpotential $\mu$-term is the dominant contribution to higgsino masses. In particular, from the dashed contour in figure 1, we conclude that ILC600 will either discover or decisively exclude models where fine-tuning is worse than $3 \%$. Precision measurements that can be made at ILC will definitively show that higgsino pair production is indeed occuring, and will allow us to measure with high precision at least the higgsino mass scale and associated mass gaps. Such a discovery would not only confirm SUSY but also strongly indicate that $W$, $Z$ and $h$ mass scales arise in a natural way via a link to nearby light higgsinos. 


\section{Acknowledgments}

We thank Peisi Huang for collaboration in the early stages of this work and we thank T. Barklow, N. Graf and J. List for discussions. This work was supported in part by the U.S. Department of Energy.

Open Access. This article is distributed under the terms of the Creative Commons Attribution License (CC-BY 4.0), which permits any use, distribution and reproduction in any medium, provided the original author(s) and source are credited.

\section{References}

[1] ATLAS collaboration, Observation of a new particle in the search for the Standard Model Higgs boson with the ATLAS detector at the LHC, Phys. Lett. B 716 (2012) 1 [arXiv: 1207.7214] [INSPIRE].

[2] CMS collaboration, Observation of a new boson at a mass of $125 \mathrm{GeV}$ with the CMS experiment at the LHC, Phys. Lett. B $\mathbf{7 1 6}$ (2012) 30 [arXiv:1207.7235] [INSPIRE].

[3] ATLAS collaboration, Search for squarks and gluinos with the ATLAS detector in final states with jets and missing transverse momentum using $4.7 \mathrm{fb}^{-1}$ of $\sqrt{s}=7 \mathrm{TeV}$ proton-proton collision data, Phys. Rev. D 87 (2013) 012008 [arXiv: 1208.0949] [INSPIRE].

[4] ATLAS collaboration, Search for squarks and gluinos with the ATLAS detector in final states with jets and missing transverse momentum and $20.3 \mathrm{fb}^{-1}$ of $\sqrt{s}=8$ TeV proton-proton collision data, ATLAS-CONF-2013-047 (2013).

[5] ATLAS collaboration, Search for squarks and gluinos in events with isolated leptons, jets and missing transverse momentum at $\sqrt{s}=8 \mathrm{TeV}$ with the ATLAS detector, ATLAS-CONF-2013-062 (2013).

[6] CMS collaboration, Search for supersymmetry in hadronic final states using MT2 in pp collisions at $\sqrt{s}=7 \mathrm{TeV}$, JHEP 10 (2012) 018 [arXiv: 1207.1798] [INSPIRE].

[7] CMS collaboration, Search for new physics in the multijet and missing transverse momentum final state in proton-proton collisions at $\sqrt{s}=8 \mathrm{TeV}$, arXiv:1402.4770 [INSPIRE].

[8] CMS collaboration, Search for supersymmetry in hadronic final states with missing transverse energy using the variables $\alpha_{T}$ and b-quark multiplicity in pp collisions at $\sqrt{s}=8$ TeV, Eur. Phys. J. C 73 (2013) 2568 [arXiv:1303.2985] [inSPIRE].

[9] M. Shifman, Frontiers Beyond the Standard Model: Reflections and Impressionistic Portrait of the conferernce, Mod. Phys. Lett. A 27 (2012) 1230043.

[10] N. Craig, The State of Supersymmetry after Run I of the LHC, arXiv:1309.0528 [INSPIRE].

[11] H. Baer, V. Barger, P. Huang, A. Mustafayev and X. Tata, Radiative natural SUSY with a 125 GeV Higgs boson, Phys. Rev. Lett. 109 (2012) 161802 [arXiv:1207.3343] [INSPIRE].

[12] H. Baer et al., Post-LHCr fine-tuning in the mSUGRA/CMSSM model with a $125 \mathrm{GeV}$ Higgs boson, Phys. Rev. D 87 (2013) 035017 [arXiv:1210.3019] [INSPIRE].

[13] H. Baer et al., Radiative natural supersymmetry: Reconciling electroweak fine-tuning and the Higgs boson mass, Phys. Rev. D 87 (2013) 115028 [arXiv:1212.2655] [INSPIRE]. 
[14] H. Baer et al., Naturalness, Supersymmetry and Light Higgsinos: A Snowmass Whitepaper, arXiv:1306.2926 [INSPIRE].

[15] H. Baer, V. Barger and D. Mickelson, How conventional measures overestimate electroweak fine-tuning in supersymmetric theory, Phys. Rev. D 88 (2013) 095013 [arXiv:1309.2984] [INSPIRE].

[16] A. Mustafayev and X. Tata, Supersymmetry, Naturalness and Light Higgsinos, arXiv: 1404.1386 [INSPIRE].

[17] H. Baer, V. Barger, D. Mickelson and M. Padeffke-Kirkland, SUSY models under siege: LHC constraints and electroweak fine-tuning, arXiv:1404.2277 [INSPIRE].

[18] K.L. Chan, U. Chattopadhyay and P. Nath, Naturalness, weak scale supersymmetry and the prospect for the observation of supersymmetry at the Tevatron and at the CERN LHC, Phys. Rev. D 58 (1998) 096004 [hep-ph/9710473] [INSPIRE].

[19] S. Akula, M. Liu, P. Nath and G. Peim, Naturalness, Supersymmetry and Implications for LHC and Dark Matter, Phys. Lett. B 709 (2012) 192 [arXiv:1111.4589] [InSPIRE].

[20] M. Liu and P. Nath, Higgs boson mass, proton decay, naturalness and constraints of the LHC and Planck data, Phys. Rev. D 87 (2013) 095012 [arXiv:1303.7472] [InSPIRE].

[21] J.L. Feng, K.T. Matchev and T. Moroi, Focus points and naturalness in supersymmetry, Phys. Rev. D 61 (2000) 075005 [hep-ph/9909334] [inSPIRE].

[22] J.L. Feng and K.T. Matchev, Focus point supersymmetry: Proton decay, flavor and CP-violation and the Higgs boson mass, Phys. Rev. D 63 (2001) 095003 [hep-ph/0011356] [INSPIRE].

[23] J.L. Feng, K.T. Matchev and D. Sanford, Focus Point Supersymmetry Redux, Phys. Rev. D 85 (2012) 075007 [arXiv:1112.3021] [INSPIRE].

[24] J.L. Feng and D. Sanford, A Natural 125 GeV Higgs Boson in the MSSM from Focus Point Supersymmetry with A-Terms, Phys. Rev. D 86 (2012) 055015 [arXiv:1205.2372] [INSPIRE].

[25] M. Perelstein and C. Spethmann, A Collider signature of the supersymmetric golden region, JHEP 04 (2007) 070 [hep-ph/0702038] [INSPIRE].

[26] M. Perelstein and B. Shakya, Fine-Tuning Implications of Direct Dark Matter Searches in the MSSM, JHEP 10 (2011) 142 [arXiv:1107.5048] [INSPIRE].

[27] M. Perelstein and B. Shakya, XENON100 implications for naturalness in the MSSM, NMSSM and $\lambda$-supersymmetry model, Phys. Rev. D 88 (2013) 075003 [arXiv:1208.0833] [INSPIRE].

[28] S.P. Martin, Compressed supersymmetry and natural neutralino dark matter from top squark-mediated annihilation to top quarks, Phys. Rev. D 75 (2007) 115005 [hep-ph/0703097] [INSPIRE].

[29] J.E. Younkin and S.P. Martin, Non-universal gaugino masses, the supersymmetric little hierarchy problem and dark matter, Phys. Rev. D 85 (2012) 055028 [arXiv:1201.2989] [INSPIRE].

[30] S.P. Martin, Non-universal gaugino masses and semi-natural supersymmetry in view of the Higgs boson discovery, Phys. Rev. D 89 (2014) 035011 [arXiv:1312.0582] [INSPIRE]. 
[31] S.F. King, M. Muhlleitner and R. Nevzorov, NMSSM Higgs Benchmarks Near $125 \mathrm{GeV}$, Nucl. Phys. B 860 (2012) 207 [arXiv:1201.2671] [INSPIRE].

[32] G. Altarelli, The Higgs: so simple yet so unnatural, Phys. Scripta T 158 (2013) 014011 [arXiv: 1308.0545] [INSPIRE].

[33] C. Brust, A. Katz, S. Lawrence and R. Sundrum, SUSY, the Third Generation and the LHC, JHEP 03 (2012) 103 [arXiv:1110.6670] [InSPIRE].

[34] H. Baer, V. Barger, M. Padeffke-Kirkland and X. Tata, Naturalness implies intra-generational degeneracy for decoupled squarks and sleptons, Phys. Rev. D 89 (2014) 037701 [arXiv:1311.4587] [INSPIRE].

[35] M. Dine, A. Kagan and S. Samuel, Naturalness in Supersymmetry, or Raising the Supersymmetry Breaking Scale, Phys. Lett. B 243 (1990) 250 [INSPIRE].

[36] A.G. Cohen, D.B. Kaplan and A.E. Nelson, The More minimal supersymmetric standard model, Phys. Lett. B 388 (1996) 588 [hep-ph/9607394] [INSPIRE].

[37] N. Arkani-Hamed and H. Murayama, Can the supersymmetric flavor problem be solved by decoupling?, Phys. Rev. D 56 (1997) R6733.

[38] T. Moroi and M. Nagai, Probing Supersymmetric Model with Heavy Sfermions Using Leptonic Flavor and CP-violations, Phys. Lett. B 723 (2013) 107 [arXiv:1303.0668] [INSPIRE].

[39] H. Baer et al., Radiatively-driven natural supersymmetry at the LHC, JHEP 12 (2013) 013 [arXiv: 1310.4858] [INSPIRE].

[40] H. Baer et al., Same sign diboson signature from supersymmetry models with light higgsinos at the LHC, Phys. Rev. Lett. 110 (2013) 151801 [arXiv:1302.5816] [INSPIRE].

[41] C. Han et al., Probing Light Higgsinos in Natural SUSY from Monojet Signals at the LHC, JHEP 02 (2014) 049 [arXiv:1310.4274] [INSPIRE].

[42] H. Baer, A. Mustafayev and X. Tata, Monojets and mono-photons from light higgsino pair production at LHC14, Phys. Rev. D 89 (2014) 055007 [arXiv: 1401.1162] [InSPIRE].

[43] P. Schwaller and J. Zurita, Compressed electroweakino spectra at the LHC, JHEP 03 (2014) 060 [arXiv:1312.7350] [INSPIRE].

[44] Z. Han, G.D. Kribs, A. Martin and A. Menon, Hunting quasidegenerate Higgsinos, Phys. Rev. D 89 (2014) 075007 [arXiv: 1401.1235] [inSPIRE].

[45] H. Baer et al., The International Linear Collider Technical Design Report - Volume 2: Physics, arXiv:1306.6352 [INSPIRE].

[46] L. Girardello and M.T. Grisaru, Soft Breaking of Supersymmetry, Nucl. Phys. B 194 (1982) 65 [inSPIRE].

[47] I. Jack and D.R.T. Jones, Nonstandard soft supersymmetry breaking, Phys. Lett. B 457 (1999) 101 [hep-ph/9903365] [INSPIRE].

[48] H. Baer et al., Physics Case for the ILC Project: Perspective from Beyond the Standard Model, arXiv: 1307.5248 [INSPIRE].

[49] H. Baer, V. Barger and P. Huang, Hidden SUSY at the LHC: the light higgsino-world scenario and the role of a lepton collider, JHEP 11 (2011) 031 [arXiv:1107.5581] [INSPIRE]. 
[50] T. Tsukamoto, K. Fujii, H. Murayama, M. Yamaguchi and Y. Okada, Precision study of supersymmetry at future linear $e^{+} e^{-}$colliders, Phys. Rev. D 51 (1995) 3153 [INSPIRE].

[51] H. Baer, R.B. Munroe and X. Tata, Supersymmetry studies at future linear $e^{+} e^{-}$colliders, Phys. Rev. D 54 (1996) 6735 [Erratum ibid. D 56 (1997) 4424] [hep-ph/9606325] [INSPIRE].

[52] H. Baer, A. Belyaev, T. Krupovnickas and X. Tata, Linear collider capabilities for supersymmetry in dark matter allowed regions of the mSUGRA model, JHEP 02 (2004) 007 [hep-ph/0311351] [INSPIRE].

[53] H. Baer, T. Krupovnickas and X. Tata, Two photon background and the reach of a linear collider for supersymmetry in WMAP favored coannihilation regions, JHEP 06 (2004) 061 [hep-ph/0405058] [INSPIRE].

[54] T. Han, S. Padhi and S. Su, Electroweakinos in the Light of the Higgs Boson, Phys. Rev. D 88 (2013) 115010 [arXiv:1309.5966] [INSPIRE].

[55] M. Berggren et al., Electroweakino Searches: A Comparative Study for LHC and ILC (A Snowmass White Paper), arXiv:1309.7342 [INSPIRE].

[56] M. Berggren et al., Tackling light higgsinos at the ILC, Eur. Phys. J. C 73 (2013) 2660 [arXiv: 1307.3566] [INSPIRE].

[57] H. Baer and J. List, Post LHC8 SUSY benchmark points for ILC physics, Phys. Rev. D 88 (2013) 055004 [arXiv: 1307.0782] [INSPIRE].

[58] H. Baer, C.-H. Chen, R.B. Munroe, F.E. Paige and X. Tata, Multichannel search for minimal supergravity at $p \bar{p}$ and $e^{+} e^{-}$colliders, Phys. Rev. D 51 (1995) 1046 [hep-ph/9408265] [INSPIRE].

[59] H. Baer, J. Ferrandis, S. Kraml and W. Porod, On the treatment of threshold effects in SUSY spectrum computations, Phys. Rev. D 73 (2006) 015010 [hep-ph/0511123] [INSPIRE].

[60] F.E. Paige, S.D. Protopopescu, H. Baer and X. Tata, ISAJET 7.69: A Monte Carlo event generator for $p p, \bar{p} p$ and $e^{+} e^{-}$reactions, hep-ph/0312045 [INSPIRE].

[61] D. Matalliotakis and H.P. Nilles, Implications of nonuniversality of soft terms in supersymmetric grand unified theories, Nucl. Phys. B 435 (1995) 115 [hep-ph/9407251] [INSPIRE].

[62] V. Berezinsky et al., Neutralino dark matter in supersymmetric models with nonuniversal scalar mass terms, Astropart. Phys. 5 (1996) 1 [hep-ph/9508249] [INSPIRE].

[63] P. Nath and R.L. Arnowitt, Nonuniversal soft SUSY breaking and dark matter, Phys. Rev. D 56 (1997) 2820 [hep-ph/9701301] [INSPIRE].

[64] J.R. Ellis, K.A. Olive and Y. Santoso, The MSSM parameter space with nonuniversal Higgs masses, Phys. Lett. B 539 (2002) 107 [hep-ph/0204192] [INSPIRE].

[65] J.R. Ellis, T. Falk, K.A. Olive and Y. Santoso, Exploration of the MSSM with nonuniversal Higgs masses, Nucl. Phys. B 652 (2003) 259 [hep-ph/0210205] [INSPIRE].

[66] H. Baer, A. Mustafayev, S. Profumo, A. Belyaev and X. Tata, Neutralino cold dark matter in a one parameter extension of the minimal supergravity model, Phys. Rev. D 71 (2005) 095008 [hep-ph/0412059] [INSPIRE].

[67] A. Sen, Tachyon Matter, JHEP 07 (2005) 065 [INSPIRE]. 
[68] H. Baer, V. Barger and D. Mickelson, Direct and indirect detection of higgsino-like WIMPs: concluding the story of electroweak naturalness, Phys. Lett. B 726 (2013) 330 [arXiv: 1303.3816] [INSPIRE].

[69] K.J. Bae, H. Baer and E.J. Chun, Mainly axion cold dark matter from natural supersymmetry, Phys. Rev. D 89 (2014) 031701 [arXiv: 1309.0519] [InSPIRE].

[70] K.J. Bae, H. Baer and E.J. Chun, Mixed axion/neutralino dark matter in the SUSY DFSZ axion model, JCAP 12 (2013) 028 [arXiv:1309.5365] [INSPIRE].

[71] H. Baer, A. Bartl, D. Karatas, W. Majerotto and X. Tata, Searching for Supersymmetry at $e^{+} e^{-}$Supercolliders, Int. J. Mod. Phys. A 4 (1989) 4111 [InSPIRE].

[72] H. Komatsu and J. Kubo, The Radiative Photino Decay Into a Higgsino, Phys. Lett. B 157 (1985) 90 [INSPIRE].

[73] H. Komatsu and J. Kubo, Decay of the light neutralino, Nucl. Phys. B 263 (1986) 265 [INSPIRE].

[74] H.E. Haber and D. Wyler, Radiative neutralino decay, Nucl. Phys. B 323 (1989) 267 [INSPIRE].

[75] H. Baer and T. Krupovnickas, Radiative neutralino decay in supersymmetric models, JHEP 09 (2002) 038 [hep-ph/0208277] [INSPIRE].

[76] E.A. Kuraev and V.S. Fadin, On Radiative Corrections to $e^{+} e^{-}$Single Photon Annihilation at High-Energy, Sov. J. Nucl. Phys. 41 (1985) 466 [InSPIRE].

[77] P. Chen, Differential luminosity under multi - photon beamstrahlung, Phys. Rev. D 46 (1992) 1186 [inSPIRE].

[78] M.E. Peskin, Consistent Yokoya-Chen approximation to beamstrahlung, SLAC-TN-04-032, LCC-0010 (1999).

[79] C. Adolphsen et al., The International Linear Collider Technical Design Report - Volume 3.II: Accelerator Baseline Design, arXiv:1306.6328 [INSPIRE].

[80] M. Drees and R.M. Godbole, Virtual photon structure functions and the parton content of the electron, Phys. Rev. D 50 (1994) 3124 [hep-ph/9403229] [INSPIRE].

[81] H. Baer and X. Tata, Weak Scale Supersymmetry, Cambridge (2006).

[82] G.A. Blair, Sparticle masses and widths via threshold scans at the linear collider, eConf $\mathbf{C}$ 010630 (2001) E3019.

[83] V.D. Barger, M.S. Berger and T. Han, Chargino mass determination at a muon collider, Phys. Rev. D 59 (1999) 71701.

[84] R.H.K. Kadala, Topics in supersymmetry phenomenology at the Large Hadron Collider, arXiv: 1205.1267 [INSPIRE].

[85] R. Kitano and Y. Nomura, Supersymmetry, naturalness and signatures at the LHC, Phys. Rev. D 73 (2006) 095004 [hep-ph/0602096] [inSPIRE]. 